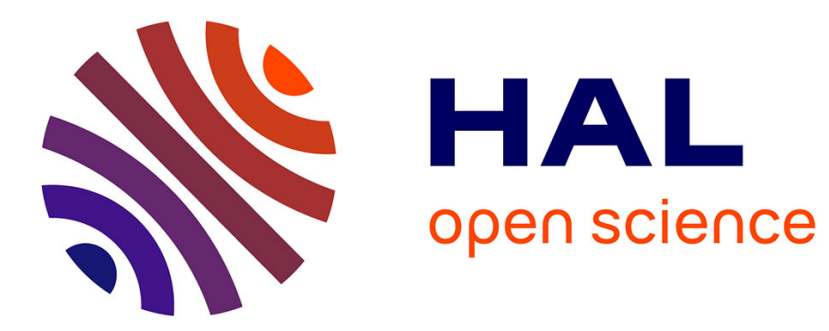

\title{
Trente ans de solitude... et de dépression
} Jean-Louis Pan Ké Shon, Géraldine Duthé

\section{- To cite this version:}

Jean-Louis Pan Ké Shon, Géraldine Duthé. Trente ans de solitude... et de dépression. Revue française de sociologie, 2013, 54 (2), pp.225-261. 10.3917/rfs.542.0225 . hal-03130790

\section{HAL Id: hal-03130790 \\ https://hal.science/hal-03130790}

Submitted on 3 Feb 2021

HAL is a multi-disciplinary open access archive for the deposit and dissemination of scientific research documents, whether they are published or not. The documents may come from teaching and research institutions in France or abroad, or from public or private research centers.
L'archive ouverte pluridisciplinaire HAL, est destinée au dépôt et à la diffusion de documents scientifiques de niveau recherche, publiés ou non, émanant des établissements d'enseignement et de recherche français ou étrangers, des laboratoires publics ou privés. 


\title{
Trente ans de solitude... et de dépression*
}

\author{
Jean-Louis PAN KÉ SHON \\ Géraldine DUTHÉ
}

\begin{abstract}
Résumé. De 1970 à 2002, la dépression a augmenté en France notamment sous les effets conjugués de l'affaiblissement de l'intégration conjugale et des modifications des règles structurant l'intériorité de l'individu. L'augmentation des divers effectifs de personnes seules a cristallisé cette moindre intégration conjugale. La crise économique qui a suivi les Trente Glorieuses, les injonctions sociales à être un soi distinct et performant sont intervenues simultanément, modifiant la régulation du sujet. En mobilisant les données relatives à la dépression et au suicide, en conjuguant les approches micro et macro, il est possible d'évaluer les effets de la progression des diverses catégories de personnes seules sur la dépression et de mieux appréhender l'impact des tensions liées à la crise économique des années 1980. L'examen en termes de chocs et d'état ordinaire du veuvage ou du chômage débouche sur l'hypothèse d'un modèle d'adaptation aux tensions, substituant une nouvelle normalité à l'ancienne, plutôt que sur celui d'une progression continue.
\end{abstract}

Mots-clés. DÉPRESSION - SOlitUde - SUICIDE - PLASTICITÉ - CRISE

L'idée d'une évolution de la société vers des formes de plus en plus pathogènes et davantage anxiogènes n'est pas nouvelle. Déjà Tocqueville constatait en 1840 que «les âmes [étaient] plus émues et plus inquiètes» chez les peuples des nouvelles démocraties qu'aux temps aristocratiques ([1840] 1951, p. 194). Près d'un siècle plus tard, Janet, à travers son expérience de psychiatre, portait un constat similaire : "La société contemporaine exige de nous des dépenses de plus en plus grandes d'énergie psychique ; [...] les choses changent vite, et il faut une tension élevée pour adapter notre action aux situations nouvelles, dans ce qu'elles ont d'original. Notre civilisation est épuisante. »(1923-1924 $\left.{ }^{1}\right)$. Plus récemment, Ehrenberg analysait les causes de la modification du sujet au cours de la seconde moitié du $\mathrm{XX}^{\mathrm{e}}$ siècle, qui ont entraîné les évolutions de la nature des tensions auxquelles le sujet doit maintenant faire face. Selon lui, trois formes régulaient auparavant les conduites : «des règles disciplinaires, qui trouvent leurs formes définitives au début du $\mathrm{XX}^{\mathrm{e}}$ siècle avec l'invention du taylorisme et du fordisme ; des règles de conformité (à l'opinion, à la tradition, à l'autorité) ; des règles d'interdiction » (2001, p. 27). Depuis, poursuit-il, l'individualité est impliquée en tant que telle. L'individu n'est plus membre d'une collectivité comme auparavant et subit une pression qui se concentre alors sur cette seule individualité. Il affirme que la «transformation de la notion de dépression se fait dans un contexte de

\footnotetext{
* Nous tenons à remercier chaleureusement les relecteurs et le comité de rédaction de la revue pour leur fructueux travail qui a permis de faire progresser ce texte.

1. Cité par Bastide (1965, p. 27).
} 
changement normatif qui devient sensible à la fin des années 1960. Effectivement, les règles traditionnelles d'encadrement des comportements individuels ne sont plus acceptées et le droit de choisir sa vie commence sinon à être la norme de la relation individu-société, du moins à entrer dans les mœurs. Au moment où la dépression se diffuse dans la médecine générale et les mœurs, la société française est en effet entrée dans sa grande transformation: elle est sortie du monde des notables et des paysans et de l'immobilité des destins de classe » (ibid., p. 34). L'univers mental des individus entre le $\mathrm{XIX}^{\mathrm{e}}$ et le $\mathrm{XX}^{\mathrm{e}}$ siècle s'est modifié : " La dépression amorce sa réussite au moment où le modèle disciplinaire de gestion des conduites, les règles d'autorité et de conformité aux interdits qui assignaient aux classes sociales comme aux deux sexes un destin ont cédé devant des normes qui incitent chacun à l'initiative individuelle en l'enjoignant à devenir lui-même. » (Ehrenberg, 1998, p. 10-11).

Ehrenberg, en s'attachant principalement au changement du régime normatif de l'individu contemporain, néglige dans le même temps que, en dehors de la régulation $^{2}$ due aux modifications des contraintes, des autorisations et des injonctions, l'intégration du sujet s'est fortement réduite, et particulièrement son intégration conjugale. La massification des personnes vivant seules ou sans conjoint est une caractéristique des sociétés contemporaines occidentales. Durkheim, au travers du suicide, a démontré la particulière nocivité de la vie sans conjoint ([1897] 1997), constat qui résiste encore plus d'un siècle après son ouvrage séminal. Dès lors, la question qui se pose est celle du candidat causal à la progression de la dépression entre 1970 et 2000. Est-ce le changement de régime normatif par les transformations de la régulation sociale du sujet notamment au travers des modifications du salariat ? Est-ce la modification structurelle de la population due à la progression de la part des personnes seules ou encore les deux à la fois et dans quelle mesure ?

La question de la progression de la prévalence dépressive prend toute sa dimension en ce qu'elle révèle des évolutions de la société qui travaillent ses membres et plus fortement certaines populations au point d'altérer leur santé mentale, voire de les pousser au suicide. Les diverses expressions de mal-être jouent alors le rôle de révélateur des tensions sociales, plus ou moins évidentes, qui traversent des individus socialement caractérisés. À l'inverse, les effets attendus de l'accentuation des tensions peuvent ne pas être au rendez-vous. Certains d'entre eux révèlent la modification au cours du temps du sens social d'un objet. Ainsi, par exemple, en passant à ce qu'autrefois il était convenu d'appeler «mère célibataire » à aujourd'hui « cheffe de famille monoparentale », il est aisé de comprendre que les tensions afférentes aient été atténuées par la transformation du regard de la société. Le chômage pourrait avoir subi cette transformation de son sens social, car les effets délétères, liés à un chômage élevé, paraissent s'être atténués au cours des années.

L'article s'attache à répondre dans une certaine mesure aux questions des impacts de la solitude et des modifications de l'emploi et du salariat en les

2. La régulation (ou réglementation) est un système de normes (du latin norma : équerre, règle) qui a cours dans une société donnée, variant en partie selon les rôles endossés par un individu donné (conjoint, père ou mère, actif occupé ou chômeur, profession, etc.). La déréglementation vient du dysfonctionnement de ce système. 
objectivant à partir de la dépression et du suicide. Dans la première partie, le contexte théorique des transformations du régime normatif est rappelé en soulignant l'adaptation des individus aux tensions charriées par les nouvelles configurations sociales. Les augmentations démographiques des parts de personnes seules sont retracées en éclairant les évolutions sociales de la seconde transition démographique dans lesquelles elles s'inscrivent. Dans la seconde partie est évaluée empiriquement la part de la prévalence dépressive due à l'affaiblissement de l'intégration conjugale, notamment par la vie solitaire au cours de la seconde moitié du $X^{\mathrm{e}}$ siècle. Pour cela, l'évolution de la dépression entre 1970 et 2002 est retracée grâce aux enquêtes décennales «Santé » et au Baromètre santé.

Sur cette période, une décomposition statistique d'un modèle non linéaire permet d'estimer au sein des évolutions ce qui relève de la progression des formes de vie sans conjoint de ce qui ressort aux autres causes. Les liens entre dépression et célibataires, veufs, séparés sont analysés. Chaque type de solitaire partage le risque commun d'être dépressif en raison de son même manque d'intégration conjugale dû à l'absence de conjoint. Au niveau micro, le différentiel de la prévalence dépressive entre catégories de solitaires est alors attribuable au choc de la séparation ou au choc du veuvage. Hors de l'impact des personnes seules et des évolutions de leur démographie, la part restante de la sur-prévalence dépressive entre 1970 et 2002 est alors attribuable aux autres causes, dont les modifications de la régulation sociale. Les liens entre dépression, activité salariée et catégories socioprofessionnelles sont examinés.

Les résultats tirés de l'examen de la dépression, cette expression singulière du mal-être à dominante féminine, demandent à être examinés par un contrepoint masculin - le suicide - à même de remettre en cause des conclusions orientées par la seule singularité de la dépression (Aneshensel, Rutter et Lachenbruch, 1991). Si la nature des données du suicide n'autorise pas des investigations micro-économétriques, en revanche les données sont disponibles sur une plus longue durée et paraissent plus stables temporellement dans leur construction qu'en ce qui concerne la dépression. Elles permettent de tester l'hypothèse de Ehrenberg de la modification du régime normatif des individus sur une période qui inclut et dépasse celle ouverte lors des années 1960.

\section{Transformations du régime normatif}

La thèse de Ehrenberg ne s'embarrasse pas de la distinction entre intégration et régulation. Il faut dire que la régulation semble être un concept tombé en désuétude, presque oublié en tout cas dans l'explication du suicide. Pourtant, Durkheim ([1897] 1997) introduit implicitement un élément réellement novateur avec la régulation. Il réside dans l'idée de l'intervention des normes sociales, même contraignantes, dans l'équilibre mental de l'individu. Ainsi, nous dit-il, l'homme en se mariant accepte de borner ses horizons à une seule partenaire sexuelle dans le cadre du mariage monogame (norme sociale) et retire de cet état une stabilité dont le bénéfice est objectivé par un moindre taux de suicide. L'immixtion du social dans l'intériorité du sujet est aussi l'une des intuitions lumineuses que nous livre Halbwachs : «Amour, haine, joie, douleur, crainte, colère ont d'abord été éprouvés et manifestés en commun, sous forme de réactions collectives. C'est 
dans les groupes dont nous faisons partie que nous avons appris à les exprimer, mais aussi à les ressentir. » (1947, p. 9). Les individus sont dotés non seulement d'un système acquis de préférences, de principes de vision, de structures cognitives et de schèmes d'action (Bourdieu, 1994), mais aussi d'un système acquis de ressenti et d'expression des affects, système intégré par l'apprentissage, par la socialisation primaire puis affiné et conforté ensuite en fonction d'une assignation sociale singulière (sexe, âge, position sociale, origine, etc.) et d'une société développant des valeurs spécifiques.

Le discours d'une intensification des sollicitations adressées aux individus, et des tensions mentales qui en résultent, est un thème récurrent à l'époque moderne. Faut-il convenir que, de génération en génération, les exigences des sociétés portant sur les individus se font continûment plus pressantes et plus personnelles ? Elias fait porter les pressions sur le processus de civilisation lui-même : «La question qui se pose est celle d'un lien possible entre le haut niveau d'efficacité que le monopole de la violence physique a atteint dans les États-nations parlementaires et la fréquence élevée des troubles psychosomatiques. » ([1988] 2010, p. 122). Mais l'essentiel ne repose peut-être pas là. En creux, il ressort que face à l'apparente accélération et à l'intensification des sollicitations, les individus finissent, bon gré, mal gré, par s'adapter à leurs nouvelles conditions d'être en société, quitte à développer de nouvelles pathologies. Baudelot et Establet soulignaient ainsi, au regard du suicide, que «le desserrement des contraintes traditionnelles ne rend pas les sociétés plus lumineuses, ni les individus plus épanouis » ([1984] 2002, p. 42). Les anciennes tensions disparaissent avec les sociétés de jadis et sont remplacées simultanément par de nouvelles pressions correspondant aux configurations sociétales modernes. Ehrenberg nous fait progresser d'un grand pas en montrant que l'individu, en s'affranchissant des anciennes règles de contrainte et d'interdiction (les anciennes normes sociales), élimine les tensions mentales qui leur étaient liées, en l'occurrence les névroses de l'interdiction au bénéfice de nouvelles pathologies.

En contrepartie, l'injonction à être un soi distinct et performant, l'adoption insensible de nouvelles normes fondées sur l'individuation du sujet, ajoutées au « déclin de la discipline au profit de l'initiative individuelle», ont laissé place à de nouvelles tensions dont le point névralgique est devenu l'insuffisance de l'individu (Ehrenberg, 1998, 2001). De fait, les idéologies et les croyances structurantes comme les certitudes religieuses et les « utopies » de gauche se sont érodées. L'insécurité des carrières, l'ébranlement des collectifs professionnels et un management plus agressif ont atomisé des salariés, devenus démunis face aux demandes de performances à la fois plus intenses et plus individualisées (Boltanski et Chiapello, 1999; Dejours et Bègue, 2009). De surcroît, l'État social, autre soutien protecteur de l'individu, tend à s'effacer, retirant ainsi un appui effectif pour les uns et potentiel pour les autres. La conjonction de ces diverses lignes de tension, l'irruption d'un individu mis à nu par l'évaporation des collectifs se sont conjuguées pour laisser un champ neuf à parcourir par un sujet en translation entre deux systèmes normatifs. La dépression, sorte de précipité moderne des mutations sociétales, cristalliserait alors la mutation du régime réglementaire du sujet au cours du XX $\mathrm{X}^{\mathrm{e}}$ siècle (Ehrenberg, 1998, 2001). Il reste alors à confirmer les analyses théoriques à partir des données de la dépression et du suicide, et à adapter l'analyse selon les rugosités de l'empirique. 


\section{Données et méthodes}

\section{Sources et mesure de la dépression}

La dépression correspond en réalité à différentes pathologies qui se hiérarchisent selon la gravité de la maladie en termes d'incapacité des individus à remplir leurs rôles sociaux ${ }^{3}$ (OMS, 1996). La dépressivité recouvre des états dépressifs souvent repérés en population générale par des outils diagnostics utilisés en épidémiologie (questionnaires utilisant des échelles de dépressivité telles que le score $C E S-D^{4}$ ). Par commodité, nous utilisons indifféremment les termes dépression et dépressivité.

Les estimations de la dépressivité varient fortement selon le protocole d'enquête, la méthode de repérage et l'indicateur adoptés. Elles n'aboutissent pas au même niveau de prévalence même pour une année proche ou identique (Le Pape et Lecomte, 1999 ; Leroux et Morin, 2006 ; Lamboy, Léon et Guilbert, 2007 ; Sapinho et al., 2008). Le but visé ici est moins de parvenir à une mesure hypothétique du niveau « réel » de la dépression que de mettre en lumière les évolutions faisant sens au regard de l'affaiblissement de l'intégration familiale et des modifications de la régulation des individus. Pour les enquêtes « Santé » de 1970 et 2002 sont considérées comme dépressives les personnes ayant déclaré un trouble lié à la dépression, soit spontanément, soit en motif de soin. Les indicateurs ainsi construits sont comparables entre les deux années.

\section{Les enquêtes «Santé»}

Les enquêtes décennales sur la santé et les soins médicaux en France métropolitaine sont mises en place depuis 1960 afin de mesurer les consommations médicales et l'état de santé des habitants. Une première visite permet de recenser les problèmes médicaux déclarés par les membres du ménage. Trois à cinq autres visites, selon les enquêtes, sont organisées pour relever les consommations médicales. Cette étude s'appuie sur la plus ancienne enquête disponible (1970) et la plus récente $(2002)^{5}$, à partir desquelles nous pouvons construire un indicateur

3. Selon la Classification internationale des maladies (Сıм-10), le sujet dépressif «présente un abaissement de l'humeur, une réduction de l'énergie et une diminution de l'activité. Il existe une altération de la capacité à éprouver du plaisir, une perte d'intérêt, une diminution de l'aptitude à se concentrer, associées couramment à une fatigue importante, même après un effort minime. On observe habituellement des troubles du sommeil et une diminution de l'appétit. Il existe presque toujours une diminution de l'estime de soi et de la confiance en soi et, fréquemment, des idées de culpabilité ou de dévalorisation, même dans les formes légères » (OMS, 2008, p. 170).

4. Le score CES-D (Center for epidemiologic studies-Depression scale) est construit grâce à vingt questions relatives à l'humeur dépressive, la tristesse, la fatigue, la perte d'énergie, les troubles de l'appétit et du sommeil, le sentiment d'infériorité, ou encore les difficultés de concentration. L'ensemble des réponses permet de positionner les personnes sur une échelle de dépressivité qui varie de 0 à 60 . À partir de 17 , une symptomatologie dépressive est suspectée ; la présence de symptômes dépressifs avérés est supposée à partir de 23.

5. L'enquête sur les dépenses de santé de 1970-1971 a été réalisée par l'Insee et le CRÉDOC auprès de plus de 7000 ménages (et plus de 23000 individus), l'enquête «Santé » de 2002-2003 a été collectée par l'Insee auprès de 17000 ménages (environ 41000 individus). La pondération n'étant pas disponible pour les données de 1970, les résultats présentés dans cet article ne sont pas pondérés, à l'exception des statistiques descriptives pour la seule enquête 2002. Les variations observées avec ou sans pondération sont faibles. 
proche à ces deux dates, ce que n'autorisaient pas les enquêtes intermédiaires de 1981 et 1991. Par construction, ces deux dernières repèrent de façon liée les consommateurs d'antidépresseurs et ceux ayant des troubles dépressifs, ce qui n'est pas le cas pour 1970 et 2002. Or, le repérage des personnes dépressives par la consommation d'antidépresseurs pose plusieurs problèmes méthodologiques. La population des consommateurs ne coïncide que partiellement avec celle des personnes dépressives ${ }^{6}$. En outre, l'augmentation de la consommation au fil des années ne peut être considérée comme le reflet de la progression de la dépression. En effet, la prescription dépend fortement des progrès pharmacologiques et des pratiques de prescription médicale (Amar et Balsan, 2004). L'impossibilité de distinguer les personnes repérées dépressives par les motifs de soins en dehors de la consommation d'antidépresseurs a conduit à écarter l'exploitation des enquêtes de 1981 et de 1991.

\section{Les indicateurs de dépressivité utilisés}

En 1970, une personne répondait pour l'ensemble des membres du ménage. L'enquête de 1970 ne disposait d'aucune question relative à la santé mentale. La dépression ne figurait pas explicitement parmi la liste des maladies proposée aux répondants, accentuant le risque de sous-déclaration. Le libellé de la question était le suivant: "Voici une liste de maladies chroniques et d'infirmités, un des membres de votre foyer est-il atteint de l'une d'entre elles ?» Vingt-deux items de maladies ou groupes de maladies étaient proposés, mais aucun ne faisait référence à une maladie mentale; seul un vingt-troisième item "Autre maladie : laquelle ? » permettait au répondant de déclarer une éventuelle maladie ne figurant pas dans cette liste, dont la dépression. Afin d'améliorer la fiabilité des informations, nous avons restreint le champ des enquêtés aux personnes âgées de plus de 18 ans ayant le statut de personne de référence ou de conjoint. Toutes les maladies déclarées ou repérées via les motifs de soins ont été codées selon une classification propre. La catégorie « syndrome dépressif » des enquêtes permet de repérer les personnes dépressives (sans que l'on puisse distinguer les déclarations spontanées des motifs de soins).

En 2002, la question utilisée était : «Avez-vous actuellement une ou plusieurs maladie(s) chroniques (i.e. une maladie qui dure depuis longtemps [ou durera longtemps] ou qui revient [reviendra] régulièrement) ? Si oui de quoi s'agit-il ?» Puis une seconde question venait compléter la première : « À part cette ou ces maladies chroniques, avez-vous actuellement d'autres maladies ou problèmes de santé ? » Ces deux questions étaient ouvertes. À la suite de la collecte, les maladies et les troubles déclarés en première visite ou repérés pendant l'enquête en motif de soins ont été classés selon la dixième version de la classification internationale des maladies (CIM-10). Afin d'approcher au plus près de la définition du « syndrome dépressif » utilisée dans l'enquête de 1970, les codes Cim que nous avons retenus concernent les troubles de l'humeur relatifs à la dépression : épisodes dépressifs (F32), troubles dépressifs récurrents (F33), troubles affectifs bipolaires (F31), troubles de l'humeur persistants (F34), troubles anxieux et

6. Les personnes dépressives peuvent ne pas consommer d'antidépresseurs (Lamboy et al., 2007) et inversement les consommateurs ne sont pas tous cliniquement considérés comme dépressifs (Amar et Balsan, 2004). 
dépressifs mixtes (F41.2). Pour optimiser la comparaison entre ces deux enquêtes, les personnes considérées dépressives sont celles l'ayant déclaré spontanément ou en motif de consultation. Cet indicateur se révèle plus restrictif que ceux employés aujourd'hui? .

\section{Le Baromètre santé}

Afin de tester l'hypothèse d'un impact différencié entre l'événement du changement de situation conjugale et l'état à plus long terme consécutif à ce changement, nous avons mobilisé les données du Baromètre santé de 2005 de l'INPES (Beck, Guilbert et Gautier, 2007), qui dispose de la date du veuvage et permet ainsi de distinguer le choc de la période ultérieure d'état de veuf. La date de la séparation n'était malheureusement pas disponible pour les séparés. De façon conventionnelle et instrumentale, nous avons retenu les dix-huit premiers mois suivant la mort du conjoint afin de disposer d'une période s'approchant d'une durée moyenne du deuil ${ }^{8}$ et de conserver des effectifs suffisants. L'indicateur de dépressivité que nous avons utilisé dans cette enquête est le score de Duke, variant de 0 à 100, calculé à partir des réponses à cinq questions sur l'état de santé mentale (Annexe 4). Plus ce score est élevé et plus la probabilité d'être dépressif est importante (Guillemin et al., 1997). L'un des avantages de l'utilisation d'un indicateur quantitatif est d'autoriser la mise en œuvre d'une régression linéaire plus robuste et ne nécessitant pas de spécification d'un seuil conventionnel de dépressivité, toujours sujet à controverse.

\section{Méthodologie d'analyse}

Il est difficile de ne pas tenir compte du genre des individus dans l'analyse de la dépression. Les manifestations pathologiques du mal-être sont reconnues pour être genrées. Ainsi, il a été successivement mis au jour des différences sexuées d'exposition aux stimuli négatifs (Gove, 1972 ; Gove et Tudor, 1973), des différentiels de vulnérabilité aux tensions (Pearlin et Schooler, 1978) et des réponses genrées aux stresseurs orientées par les structures de l'intériorité construites différemment pour chaque sexe lors de la socialisation primaire (Coster, 2005 ; Cousteaux et Pan Ké Shon, 2008). Il y a donc une nécessité à évaluer les évolutions de la prévalence dépressive en fonction du sexe des individus d'autant qu'elle est deux fois plus importante pour les femmes. Les modèles logistiques de la dépression évaluent simultanément les risques des hommes et des femmes en croisant chaque variable indépendante avec le sexe. L'introduction d'une indicatrice supplémentaire du sexe de l'individu dans les régressions neutralise son effet spécifique. Autrement dit, les variables hommes-femmes sont débarrassées de l'écart initial redevable au genre.

7. Actuellement, le repérage de la dépression dans les enquêtes en population générale est réalisé plus ou moins indirectement grâce à une échelle de dépressivité dont les échelons sont établis au moyen d'un score calculé à partir de plusieurs questions (souvent de 5 à 20) mettant au jour la présence de symptômes dépressifs (par exemple, le score $C E S-D$ ou celui de Duke).

8. Nous nous appuyons sur le constat de Bowlby selon lequel la durée de l'affliction du deuil est variable mais dépasse un an en moyenne ([1984] 2008, p. 133-137). 
Le niveau de repérage de la dépression dépend du protocole d'interrogation, des nomenclatures de classification du trouble, de la connaissance des symptômes et de leur degré de dicibilité sociale. L'individu interrogé doit se savoir atteint de la maladie pour la déclarer. Or, avec les progrès médicaux, le développement de la prévention et de la prise en charge des problèmes de santé, la connaissance et le diagnostic des maladies ont évolué au cours des trente ans séparant nos deux enquêtes. Différents biais sont ainsi associés à la mesure d'une maladie entre deux dates éloignées (Sermet et Cambois, 2002), surtout s'agissant de troubles mentaux à cause des représentations populaires et négatives de "la folie ». Les statistiques descriptives de la dépression n'échappent pas à ces biais.

Toutefois, un moyen instrumental de contourner en partie ce problème est de mettre en œuvre la décomposition statistique de Blinder-Oaxaca modifiée pour les modèles non linéaires par Fairlie (2005) (Annexe 1). Cette méthode économétrique permet, dans le calcul de l'évolution de la dépression, de séparer ce qui revient à la progression des effectifs des personnes seules, de ce qui ressortit aux autres causes modifiant les niveaux de prévalence de la dépression à une date donnée. Il est alors possible d'évaluer « toutes choses étant égales par ailleurs » la part redevable au manque d'intégration due à la seule progression des effectifs des solitaires débarrassée des effets spécifiques de chacune des périodes. Ce traitement économétrique revient à indifférencier les impacts de la dépression aux deux dates retenues, car les coefficients de la régression sont issus des deux enquêtes empilées. Les variations des contributions à l'augmentation de la dépression proviennent de la substitution variable après variable de la distribution de 1970 par celle de 2002 .

\section{Que mille solitaires s'épanouissent...}

Partant de l'observation du plus grand nombre de suicidés parmi les célibataires, les divorcés et les veufs par rapport aux personnes mariées, Durkheim, s'inquiétant de la direction prise par la société, se prononçait contre le divorce. Peine perdue. L'après-Seconde Guerre mondiale a vu fleurir les intermittences du couple ainsi que la vie solitaire ou sans conjoint. Dans les pays occidentaux, la décennie 1965-1975 constitue une période charnière où l'intimité des individus et leurs comportements individuels sont profondément retravaillés. Ces bouleversements sont notamment objectivés par une rupture des tendances démographiques et culturelles, marquant le début de ce que certains ont appelé « la seconde transition démographique » (Lesthaeghe, 1995). Ainsi, la fécondité diminue, la taille de la famille se réduit et s'homogénéise, la cohabitation et le nombre de naissances hors mariage s'amplifient, l'âge au premier mariage s'élève, le taux de personnes mariées s'amenuise, les ruptures d'union et le divorce se diffusent, la vie ou les épisodes de vie solitaires se multiplient. Ces modifications démographiques interviennent au moment d'une conjonction économique, sociale et culturelle exceptionnelle. La société est en profonde mutation avec deux caractéristiques majeures : l'autonomisation de l'individu et l'émancipation des femmes (ibid.).

Cette période de mutation se caractérise par l'arrivée massive des baby-boomers à l'âge adulte, dans un contexte économique extrêmement favorable de quasi- 
plein-emploi et d'augmentation des standards de confort des familles. Cette arrivée de ces générations de jeunes s'est accompagnée d'une contestation idéologique du triptyque institutionnel et intégrateur Travail-Famille-Patrie dédramatisant la vie solitaire et d'une mise en accusation de la famille «bourgeoise». Par ailleurs, les femmes se sont autonomisées grâce à la synergie créée par une éducation scolaire plus élevée, induisant des exigences nouvelles envers leur(s) partenaire(s), et par la montée de l'emploi salarié féminin, autorisant une certaine indépendance financière. En investissant progressivement et massivement le marché du travail dans la seconde moitié du $\mathrm{XX}^{\mathrm{e}}$ siècle, les femmes se sont simultanément exposées aux tensions intrinsèques au salariat, particulièrement celles que subissent les salariés aux positions modestes. À cela s'est ajoutée une meilleure maîtrise des naissances que par le passé, permettant un meilleur contrôle de leur calendrier de fécondité. De plus, la diffusion des idées féministes a permis de porter un regard neuf et décomplexé sur une autonomie féminine auparavant douteuse (Kaufmann, 1999). Depuis 1960, l'espérance de vie s'est allongée et les écarts de mortalité se sont creusés entre les sexes, renforçant les effectifs de femmes âgées qui, conséquemment, sont amenées à vivre plus souvent seules que les hommes (Gaymu et al., 2008), d'autant que leur remise en couple est moins aisée que chez ces derniers (Cassan, Mazuy et Clanché, 2001).

Sous la conjonction d'exigences nouvelles, que traduisent et autorisent ces modifications économiques, démographiques et culturelles, l'intégration conjugale s'est affaiblie, les couples sont devenus plus rares et plus contractuels. La famille moderne se caractérise mieux par l'individualisation, la qualité des liens relationnels et son étatisation (Singly, 2007). Rien qu'entre les recensements français de 1962 et 2005, les personnes vivant seules sont progressivement passées de $6 \%$ à plus de $14 \%$ de la population française (Tableau 1), et même 16,2 \% des personnes de 15 ans et plus en 2008 (Insee, 2012). Dans cette période, la proportion des familles monoparentales a plus que doublé et représente actuellement 8,1\% des ménages français. Aujourd'hui, 41,4\% des ménages sont constitués de personnes vivant seules ou sans conjoint. Cette relative masse de célibataires, de séparés, de veufs et de monoparents représente des populations particulièrement perméables au mal-être, quelles que soient les formes qu'il puisse prendre pour s'exprimer : suicide, risque suicidaire, dépression, dépendance alcoolique, sentiment de solitude, etc. (Cousteaux et Pan Ké Shon, 2008). Dès lors, la forte augmentation des effectifs de personnes seules devrait logiquement s'accompagner d'une progression corrélative des expressions du mal-être.

Mais parler des personnes seules comme si elles constituaient une entité propre ne permet pas de saisir leurs singularités. Effectivement, chaque catégorie de personnes seules, célibataires, séparées, veuves scande tendanciellement trois moments distincts du cycle de vie, dessinant à gros traits la jeunesse, la maturité, la vieillesse. Elles forment des groupes aux proportions inégales. Au recensement de 1999, parmi les 9,1 millions de Français vivant sans conjoint dans leur logement, $37 \%$ étaient célibataires, $27 \%$ veufs, $12,5 \%$ divorcés, 4,5\% mariés et $19 \%$ monoparents. Depuis le recensement de 1982, l'augmentation du nombre de personnes seules était pour près de $60 \%$ due à la progression du nombre de célibataires et pour $20 \%$ à celle des divorcés (Pan Ké Shon, 2002). 
TABLEAU 1. - Évolution de la part des personnes vivant seules en France (en \%)

\begin{tabular}{|l|r|r|r|r|r|r|c|}
\cline { 2 - 7 } \multicolumn{1}{c|}{} & 1962 & 1968 & 1975 & 1982 & 1990 & 1999 & 2005 \\
\hline Personnes vivant seules $^{\mathrm{a}}$ & 6,3 & 6,6 & 7,7 & 9,1 & 10,6 & 12,9 & 14,2 \\
\hline $\begin{array}{l}\text { Ménages de personnes } \\
\text { vivant seules }\end{array}$ & 16,6 & 20,4 & 22,2 & 24,5 & 27,2 & 31,0 & 32,9 \\
\hline $\begin{array}{l}\text { Ménages de familles } \\
\text { monoparentales }\end{array}$ & - & 2,9 & 3,0 & $3,6^{\mathrm{b}}$ & 6,6 & 7,4 & 7,6 \\
\hline
\end{tabular}

Champ : Individus et ménages ordinaires.

Source : Recensements de la population, enquête annuelle de recensement 2005, Insee. Calculs des auteurs. ${ }^{a}$ Individus vivant en ménages ordinaires de France métropolitaine.

${ }^{\mathrm{b}}$ Depuis 1982, les enfants de plus de 25 ans sont pris en compte dans l'établissement d'une famille monoparentale.

En toute rigueur, le manque d'intégration conjugale, que partagent les célibataires, les séparés et les veufs, devrait se cristalliser à un même degré dans une expression de mal-être, ici la dépressivité. Concrètement, ce manque d'intégration conjugale vient de l'impossibilité d'alléger les tensions de la vie quotidienne par le conjoint. Il y a à la fois un déficit «structurel » de support social et des interactions limitées en fréquence et en durée (Hughes et Gove, 1981). Or, comme nous allons le vérifier, il s'observe bien des niveaux de dépressivité différenciés entre ces trois catégories de solitaires. Il faut donc reconnaître que cet écart est redevable à un autre phénomène que l'intégration conjugale puisque chacune de ces trois catégories vit également sans conjoint. Rappelons que ce ne peut être la présence d'enfants qui explique ces différentiels puisqu'il a pu être montré que les enfants n'ont pas d'effet protecteur sur la dépression comme sur d'autres expressions de mal-être (Cousteaux et Pan Ké Shon, 2008). Implicitement, les personnes sans conjoint incorporent des perspectives de vie dont les horizons les éloignent les unes des autres car l'injonction sociale à se réaliser par le couple n'a évidemment pas la même acuité pour les célibataires, les veufs et les divorcés. Pourtant, le moindre poids du regard social porté sur ces états de célibat, de veuvage, de séparation, de divorce ne s'est pas accompagné d'une moindre nocivité de la vie solitaire. Une hypothèse alternative partirait plus simplement de l'absence de choc du veuvage ou de la séparation pour les célibataires. Les différentiels de prévalence dépressive seraient alors dus non pas à l'état de personne sans conjoint, mais à l'événement ou au choc de la séparation et de la mort du conjoint.

\section{Augmentation conjointe de la dépression et des personnes seules entre 1970 et 2002}

\section{Progression de la dépression}

La dépression touche près de trois fois plus les femmes que les hommes (Tableau 2). C'est ce qui est globalement observé quels que soient les enquêtes et les indicateurs utilisés (Leroux et Morin, 2006 ; Sapinho et al., 2008). Le triplement du taux de dépressivité entre 1970 et 2002 tend à conforter la thèse d'une société du labeur davantage pathogène à cause de l'individualisation du sujet 
(Ehrenberg, 1998). Ces constatations sont aussi étayées par d'autres analystes relevant les modifications du management des salariés depuis les années 1980-1990 (Beaud et Pialoux, 2003 ; Dejours et Bègue, 2009). Ces transformations correspondent bien aux aspects négatifs mis à plat dans le modèle de stress au travail de Karasek et Theorell (1990) : forte demande psychologique, faible autonomie du salarié, moindre support social tiré des collègues à cause de leur mise en compétition. Boltanski et Chiapello (1999) avaient déjà constaté les profondes modifications du management des salariés, justifiant selon eux l'expression d'un «nouvel esprit du capitalisme ». Nos résultats ne viennent donc pas en contradiction avec ces propos puisque la dépression augmente bien pour les hommes jusqu'à la soixantaine puis baisse aux âges où l'on accède massivement à la retraite en France. Pour les femmes, la progression de la dépression est bien plus spectaculaire et s'accorde avec leur investissement massif du marché du travail au cours de ces trois décennies. Si les évolutions de la prévalence dépressive entre 1970 et 2002 sont sujettes à caution, à cause des remarques émises précédemment (voir Données et méthodes), les écarts hommes-femmes restent pertinents, ces deux populations étant soumises aux mêmes biais potentiels. On observe que, là où les courbes étaient globalement parallèles entre les sexes, en 2002, il y a bien eu un accroissement féminin de la dépressivité aux environs de trente ans jusqu'à la soixantaine incluse (Figure 1).

\section{TABLEAU 2. - Proportion de personnes dépressives selon le sexe (en \%)}

\begin{tabular}{|c|c|c|c|}
\cline { 2 - 4 } \multicolumn{1}{c|}{} & Enquête de 1970 & Enquête de 2002 & Ratio 2002/1970 \\
\hline Hommes & 0,6 & 2,3 & 3,8 \\
\hline Femmes & 1,8 & 6,0 & 3,3 \\
\hline Ensemble & 1,3 & 4,2 & 3,2 \\
\hline
\end{tabular}

Note : Personnes repérées dépressives.

Champ : Personnes de référence et conjoint-e-s âgé-e-s d'au moins 18 ans.

Source : Enquêtes « Santé » de 1970 et 2002.

Les femmes sont plus souvent employées et occupent des postes où les demandes peuvent être fortes et l'autonomie limitée, deux caractéristiques délétères pour la santé mentale. Ainsi, le rapport de l'OCDE sur la santé mentale note : «Ces emplois associent, plus souvent que dans d'autres professions, exigences cognitives élevées et faible latitude décisionnelle - une combinaison susceptible de générer une situation de tension au travail, c'est-à-dire un stress professionnel malsain, qui est un facteur reconnu de mauvaise santé mentale. » (OCDE, 2012, p. 223). Toutefois, la dépression féminine culmine entre 50 et 70 ans, âges où les taux d'activité professionnelle sont faibles ${ }^{9}$. Ce constat interroge la partie de la thèse de Ehrenberg portant sur l'individualisation des salariés qui se serait traduite

9. Certaines divergences s'observent entre notre indicateur et celui construit à partir d'un score de dépressivité $(C E S-D)$. La baisse de la dépression après 70 ans est constatée à partir de notre indicateur, alors qu'à l'inverse le $C E S-D$ indique que la prévalence dépressive progresse fortement après 65 ans pour les femmes et dans une moindre mesure pour les hommes (Cousteaux et Pan Ké Shon, 2008). Seule une étude méthodologique approfondie pourrait expliquer ces divergences. Pour notre part, nous relevons que le vieillissement, en dehors même de la dépression, est lié à quatre items au moins du score CES-D : les manques d'appétit, de concentration, de sommeil et d'entrain. 
par de plus nombreuses dépressions, puisque le fort accroissement porte sur les femmes et intervient davantage parmi les populations inactives... Néanmoins, les résultats ne le contredisent pas formellement, puisque la dépression semble s'accroître à tous les âges, actifs y compris.

\section{FIGURE 1. - Proportions de personnes dépressives}
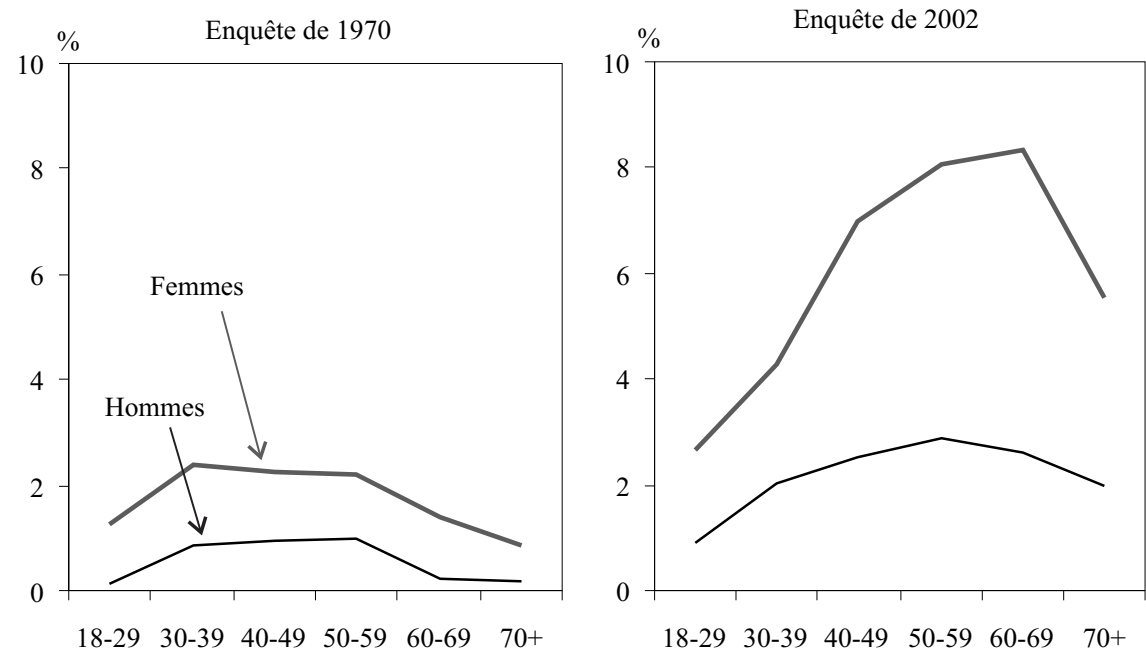

Champ : Personnes de référence et conjoint-e-s âgé-e-s d'au moins 18 ans.

Source : Enquêtes « Santé » 1970 et 2002.

\section{Personnes seules, dépression et biais de sélection}

La forte progression de la vie solitaire dénote des bouleversements profonds dans les rapports aux autres et particulièrement dans les relations d'intimité. Les contraintes institutionnelles sont moindres qu'autrefois, les exigences réciproques des membres du couple sont supérieures, et les épisodes de solitude conséquemment plus fréquents. Cependant, dans la relation vie solitaire et dépression, il subsiste le soupçon d'un possible biais de sélection par lequel les dépressifs seraient handicapés dans la formation d'un couple ou que leur union serait plus fragile pour ceux l'ayant formé. Dès lors, ce ne serait pas les personnes seules qui seraient plus perméables à la dépression, mais les dépressifs qui seraient plus souvent seuls. Les études anglo-saxonnes à partir de données longitudinales indiquent que la sélection matrimoniale liée à l'état de santé mentale est soit limitée (Waldron, Hughes et Brooks, 1996), soit inexistante (Simon, 2002). L'étude d'une cohorte de jeunes adultes met en évidence les chances identiques de se marier des dépressifs des deux sexes. Seuls les alcooliques sont davantage écartés du marché matrimonial (Horwitz et White, 1991). Les ruptures matrimoniales montrent que les personnes mariées qui se sont par la suite séparées rapportent plus de dépression et de problèmes d'alcool avant la rupture que ceux qui sont restés mariés (Simon, 2002). Cependant, la circularité de la relation (mésentente-dépressionrupture-dépression) rend la recherche de la cause initiale illusoire (ibid.). 
D'autres remarquent qu'aucune preuve n'a été jusqu'à présent fournie pour étayer ce soupçon déjà ancien de biais de sélection (Hughes et Gove, 1981). A contrario, ils s'attachent à souligner les qualités préservatives et singulières $\mathrm{du}$ conjoint, dont les solitaires sont privés : les interactions plus nombreuses qu'avec tout autre partenaire, souvent quotidiennes, les durées plus importantes, l'engagement plus intense, les relations mutuelles d'obligation et de soutien qui aboutissent logiquement à une intégration plus forte. Ce que d'autres résument par les supports émotionnel et instrumental (par exemple, Jong-Gierveld et Tilburg, 1987). De surcroît, le support social se lit comme un élément de préservation ou d'acquisition de l'estime de soi par la valorisation de soi au travers des relations interpersonnelles privilégiées (Turner et Turner, 1999). La mise à mal de l'estime de soi favorise des pathologies mentales dont la dépression (Bibring, 1953; Jacobson, 1971 ; Fredén, 1983 ; Tousignant, 1992). Enfin, le veuvage récent est un événement aléatoire qui échappe à l'effet de sélection des personnes dépressives et de la plus grande difficulté à la formation ou à la durabilité du couple. Le fait est que les personnes veuves sont non seulement plus souvent dépressives dans les mois suivant le décès du conjoint, mais aussi lorsque le choc s'est éloigné dans le temps.

\section{Progression des personnes seules}

Les personnes vivant seules ou sans conjoint - célibataires, monoparents, veufs, séparés - composent un groupe hétérogène avec des vécus et des perspectives de vie qui les éloignent les unes des autres plus qu'ils ne les rapprochent. En 1970, les veufs constituaient presque la moitié des personnes seules, mais progressivement les célibataires sont devenus le groupe dominant (Tableau 3). Aujourd'hui, les enfants ne décohabitent plus de chez les parents pour s'installer en couple comme autrefois. La période qui s'ouvre après avoir quitté le nid familial est celle des expériences, souvent revendiquées comme telles (Van de Velde, 2004). Cette nouvelle «liberté », ces diverses expériences de construction de soi peuvent se révéler plus déstabilisantes qu'auparavant et conséquemment s'exprimer par des pathologies mentales. Simultanément, les parts des personnes séparées et des chefs de familles monoparentales ont fortement progressé, plus modérément pour celle des veufs, favorisant l'augmentation de la prévalence dépressive.

\section{TABLEAU 3. - Proportion et répartition de personnes vivant sans conjoint selon les enquêtes "Santé » (en \%)}

\begin{tabular}{|l|c|c|c|c|}
\cline { 2 - 5 } \multicolumn{1}{c|}{} & \multicolumn{2}{c|}{ Enquête de 1970} & \multicolumn{2}{c|}{ Enquête de 2002} \\
\cline { 2 - 5 } \multicolumn{1}{c|}{} & Proportion & Répartition & Proportion & Répartition \\
\hline Célibataire & 3,2 & 24,7 & 7,9 & 33,7 \\
\hline Veuf & 5,9 & 46,4 & 6,8 & 28,9 \\
\hline Séparé & 1,1 & 8,3 & 3,9 & 16,5 \\
\hline Monoparent de plus de 65 ans & 0,9 & 6,9 & 0,5 & 2,0 \\
\hline Ensemoparent de moins de 65 ans & 1,8 & 13,7 & 4,4 & 18,9 \\
\hline
\end{tabular}

Champ : Personnes de référence âgées d'au moins 18 ans.

Source : Enquêtes « Santé » 1970 et 2002. 


\section{Relations entre dépression et personnes seules}

Afin de mettre en lumière les liens entre dépression et vie solitaire débarrassés des effets des autres caractéristiques sociodémographiques, un modèle logistique estimant le risque d'être dépressif est utilisé. Le modèle est contrôlé par le sexe, la situation de l'individu dans le ménage, le groupe d'âge, l'occupation professionnelle et le niveau de diplôme. Chaque caractéristique est croisée avec le sexe afin de tenir compte de la nature genrée de l'indicateur et de repérer des effets différenciés entre hommes et femmes. Une indicatrice du sexe des répondants est introduite, elle permet de réduire le différentiel initial de dépressivité entre hommes et femmes.

En 1970, les hommes seuls célibataires et les femmes âgées de moins de 65 ans vivant seules avec enfant(s) constituaient les seuls groupes pour lesquels le risque d'être dépressif était significativement plus élevé par rapport aux personnes en couple avec enfant(s) (Tableau 4). Dans l'ensemble, les résultats masculins pour 1970 ne sont pas significatifs, car ils pâtissent d'effectifs de dépressifs insuffisants. Trente ans plus tard, à l'exception des monoparents de 65 ans ou plus, toutes les personnes vivant sans conjoint - quels que soient leur sexe, leur statut matrimonial et la charge d'enfant(s) - présentent des risques de dépressivité significativement plus élevés que les personnes en couple avec ou sans enfant(s). En trente ans, non seulement le nombre des personnes seules s'est accru, mais leur risque de dépressivité s'est accentué.

TABLEAU 4. - Extrait des modèles logistiques du risque d'être dépressif

\begin{tabular}{|c|c|c|c|c|c|c|c|c|}
\hline & \multicolumn{4}{|c|}{ Enquête de 1970} & \multicolumn{4}{|c|}{ Enquête de 2002} \\
\hline Intercept & \multicolumn{4}{|c|}{$-4,3 * * *$} & \multicolumn{4}{|c|}{$-4,3 * * *$} \\
\hline \multirow[t]{2}{*}{ Sexe } & \multicolumn{4}{|c|}{0,3} & \multicolumn{4}{|c|}{$1,5 * * *$} \\
\hline & \multicolumn{2}{|c|}{ Hommes } & \multicolumn{2}{|c|}{ Femmes } & \multicolumn{2}{|c|}{ Hommes } & \multicolumn{2}{|c|}{ Femmes } \\
\hline Célibataire & 1,2 & $* *$ & 0,2 & & 1,1 & $* * *$ & 0,8 & $* * *$ \\
\hline Veuf & 1,1 & & $-0,1$ & & 1,5 & $* * *$ & 0,7 & $* * *$ \\
\hline Séparé & $-12,2$ & & 1,0 & & 1,2 & $* * *$ & 1,1 & $* * *$ \\
\hline Monoparent de 65 ans ou plus & $-10,9$ & & 0,1 & & 1,6 & & $-0,1$ & \\
\hline Monoparent de moins de 65 ans & $-12,3$ & & 1,0 & $* * *$ & 1,3 & $* * *$ & 0,8 & $* * *$ \\
\hline Couple avec enfant(s) & Réf. & & Réf. & & Réf. & & Réf. & \\
\hline Couple sans enfant & 0,0 & & 0,1 & & 0,4 & $*$ & 0,2 & \\
\hline Autre & $-0,2$ & & $-0,6$ & & 0,2 & & $-0,4$ & \\
\hline
\end{tabular}

Note: $* * * \mathrm{p}<1 \%$; ** $\mathrm{p}<5 \%$; $\mathrm{p}<10 \%$.

Un modèle de régression logistique est établi par enquête, chaque variable des modèles est croisée avec le sexe (extrait du modèle complet présenté en Annexe 2).

Champ : Personnes de référence et conjoint-e-s âgé-e-s d'au moins 18 ans.

Source : Enquêtes «Santé » 1970 et 2002.

Les résultats récents obtenus à partir d'indicateurs de dépressivité différents montrent qu'au regard de la dépression le veuvage semble la forme la plus délétère des trois catégories de solitaires pour les hommes (Tableau 4 et Cousteaux et Pan Ké Shon, 2008). Les situations vécues de solitude n'ont ni la même signification, ni les mêmes conséquences pour les hommes et les femmes (Umberson, 
Wortman et Kessler, 1992). Le départ du conjoint libère les femmes d'un surcroît de tâches ménagères, quand à l'inverse les hommes doivent assumer de nouvelles activités d'autant plus pénibles qu'ils les ont plus rarement pratiquées par le passé. Pourtant, la moindre familiarité avec les tâches domestiques semble une explication insuffisante puisque les hommes séparés, qui connaissent une charge et des conditions semblables, sont moins pénalisés par la dépression que les veufs. C'est surtout qu'après la rupture, et on suppose par extension après la mort du conjoint, les femmes pâtissent de la réduction de leur niveau de vie et les hommes de la solitude (Gerstel, Riessman et Rosenfield, 1985 ; Umberson, Wortman et Kessler, 1992 ; Fokkema et Dykstra, 2002).

La surpénalité à la solitude des veufs par rapport aux autres situations de solitude ne provient pas du seul manque de conjoint, car ils partagent cette situation avec les célibataires et les séparés. On pourrait avancer d'autres hypothèses afin d'expliquer cette surpénalité. 1) Les hommes prendraient conscience au moment du veuvage, à tort ou à raison, qu'une remise en couple est peu probable. Cette perspective leur serait davantage délétère, contrairement aux femmes, qui auraient déjà intériorisé ce fait, le marché de l'union à ce moment de leur vie leur étant défavorable. 2) Dans une vision féministe et schématique, la perte du conjoint n'est pas équivalente pour les deux sexes : le veuvage des femmes entraîne la perte d'un conjoint dominant, quand le veuf perd une conjointe dominée. Il est alors compréhensible que la perte du conjoint soit davantage délétère pour les hommes et se cristallise dans une plus forte prévalence dépressive. Toutefois, ces hypothèses raisonnées ne sont pas acquises, comme nous allons le constater.

\section{Chocs et états du veuvage}

Il se déduit du différentiel du risque d'être dépressif entre chaque type de solitaire qu'il ne repose pas sur le manque d'intégration conjugale. Effectivement, célibataires, séparés, monoparents et veufs partagent cette même expérience de l'absence du conjoint intégrateur. Il faut donc chercher une autre dimension pour expliquer ces différentiels. Si tous les veufs ${ }^{10}$ subissent la perte du conjoint, les séparations, quant à elles, sont fréquemment à l'initiative d'un seul membre du couple, et ce dernier ne subit pas avec la même intensité la toxicité de la rupture. On pourrait penser que, touchant globalement un séparé sur deux, leurs prévalences dépressives seraient logiquement bien moindres que celles observées chez les veufs, qui, eux, subissent tous la séparation avec le conjoint décédé. En réalité, les combinaisons entre initiative de la rupture et réactions des conjoints sont plus complexes. La séparation peut aussi bien venir d'une dégradation insensible des rapports, représenter une situation satisfaisante et une issue logique pour chacun des conjoints, voire être l'occasion d'une libération ou d'un renouveau, qu'être vécue sur le mode de la trahison, de l'angoisse de la solitude et de l'amputation des revenus (Aseltine et Kessler, 1993 ; Kaufmann, 1999 ; Singly, 2011). Les situations de rupture sont donc plus hétérogènes que celles du veuvage.

Dans le veuvage, il est nécessaire de différencier deux phénomènes : le choc ressenti au cours de la période couvrant quelques mois après l'événement, et

10. Il serait plus juste de parler de «veuves », le veuvage étant à $85 \%$ féminin. 
l'état, qui, lui, correspond à l'état ordinaire de la vie sans le conjoint décédé en dehors de l'événement du choc. On peut penser que le choc du veuvage est constitué de divers éléments. Le choc émotionnel créé par le deuil, le sentiment d'irrémédiable et la perturbation due au «changement brusque de l'univers social de référence » (Besnard, 1987, p. 103). L'anomie durkheimienne, appréhendée comme la perte de repères, connaît schématiquement un régime en deux temps. Elle constitue un élément de déstabilisation violent le temps du choc, puis se stabilise avec le temps. On retrouve ici une illustration de la distinction entre «anomie aiguë » et « anomie chronique » (Durkheim, [1897] 1997) ou « anomie de crise »versus « anomie institutionnalisée », selon les termes de Besnard (1987, p. 102).

La dynamique temporelle du veuvage permet de mieux appréhender les imbrications entre intégration et régulation. Le choc du veuvage relève d'une dérégulation «aiguë», d'une brusque déstabilisation due au choc émotionnel, d'une reconfiguration violente du paysage mental de l'individu par la perte du conjoint. À cela s'ajoute la puissante déstabilisation due à la perte des repères, à l'absence de structuration de l'individu par les petites activités quotidiennes réalisées auparavant en fonction du partenaire, par l'absence de ce cadre référent contraignant mais structurant. Ces sentiments et ce choc s'atténuent progressivement au fil du temps, ce qu'illustre l'adage populaire « Le temps panse les plaies ». La déstabilisation provenant de la perte de structuration de l'individu par les activités quotidiennes serait particulièrement sensible au cours de la période de choc. De fait, aux habitudes anciennes vont s'en substituer insensiblement d'autres, d'autres façons de réaliser les menues tâches journalières, aboutissant à une nouvelle structuration du temps. Cependant, la régulation qui prévalait est devenue plus flottante et le cadre des contraintes conjugales s'est relâché à celui que l'individu lui-même s'impose, en fonction bien sûr des normes et valeurs intégrées. Cette perturbation n'est pas uniquement redevable au vertige des possibles cher à Durkheim (Besnard, 1987), mais aussi à l'absence de support social fourni auparavant par le conjoint, dont la présence protégeait, rassurait, permettait d'évacuer les tensions, de communiquer sur les choses de la vie et au final renvoyait l'importance qu' ego revêtait aux yeux de cet autre particulièrement significatif, ce qu'en termes durkheimiens on nomme intégration. En ce sens, l'état de dérégulation conjugale ou « anomie chronique » correspond à un manque d'intégration conjugale.

Deux résultats importants sont tirés de l'exploitation empirique. Confirmant l'intuition, le choc du veuvage possède bien un impact plus puissant sur la santé mentale que l'état ordinaire de veuf, et cela indifféremment du genre (Tableau 5). Deuxièmement, il n'y a pas de différence significative entre le degré de dépressivité des célibataires et des personnes en état de veuvage, et ce quel que soit le sexe. Au regard de la dépression, l'état de veuvage paraît posséder la nocivité du célibat. Autrement dit, c'est bien le manque commun de conjoint, le déficit d'intégration conjugale des célibataires et de l'état de veufs qui les rapproche. Ces constats demanderaient à être consolidés à partir d'autres études. On peut faire l'hypothèse que, si les données permettaient de distinguer dans la séparation, d'une part, le choc de l'état et, d'autre part, le membre du couple initiateur de celui qui subit la séparation, elles révèleraient des tendances similaires, bien que les situations de ruptures soient plus diverses et complexes (Kaufmann, 1999; Singly, 2011). On retiendra que les progressions des effectifs de veufs, de séparés et aussi de monoparents s'accompagneront corrélativement d'une augmentation de la dépression et que l'impact du fort développement du célibat aura une incidence plus limitée au cours des trente années d'intérêt. 
TABLEAU 5. - Extrait de la régression linéaire du risque d'être dépressif

\begin{tabular}{|l|c|c|c|c|}
\cline { 2 - 5 } \multicolumn{1}{c|}{} & \multicolumn{4}{c|}{ Baromètre santé 2005 } \\
\hline Constante & \multicolumn{4}{c|}{$19,7 * * *$} \\
\hline Sexe & \multicolumn{2}{|c|}{ Hommes } & \multicolumn{2}{c|}{ Femmes } \\
\hline Célibataire seul & 6,0 & $* * *$ & 2,5 & $* * *$ \\
\hline Séparé seul & 4,2 & $* * *$ & 3,3 & $* * *$ \\
\hline Veuf seul, état & 6,2 & $* * *$ & 2,9 & $* * *$ \\
\hline Veuf seul, choc & 8,8 & $*$ & 6,8 & $* * *$ \\
\hline Monoparents & 3,6 & $* *$ & 2,0 & $* * *$ \\
\hline Couples & Réf. & - & Réf. & - \\
\hline
\end{tabular}

Note: $* * * \mathrm{p}<1 \%$; ** $\mathrm{p}<5 \% ; * \mathrm{p}<10 \%$.

L'indicateur de dépressivité est construit grâce au score de Duke (Annexe 4). Les résultats viennent d'un seul modèle de régression linéaire, où les variables sont croisées avec celles du sexe. Le modèle est contrôlé par le sexe, l'âge, les diplômes, le statut d'activité, les revenus, les événements importants pendant l'enfance et l'année écoulée (modèle complet disponible auprès des auteurs).

Champ : Individus de 12 à 75 ans.

Source : Baromètre santé 2005 (INPES). Exploitation par les auteurs.

\section{Impact de la progression des personnes seules sur la dépression}

La décomposition statistique proposée par Blinder-Oaxaca étendue aux modèles logistiques par Fairlie (2005) autorise l'évaluation des contributions propres de chaque variable à l'évolution de la prévalence dépressive entre 1970 et 2002, tout en contrôlant les autres variables du modèle. Elle s'attache aux évolutions de la structure de la population et notamment à la progression des effectifs des personnes seules sur les risques d'être dépressif (Annexe 1). L'établissement de modèles identiques à des fins de strictes comparaisons entre 1970 et 2002 contraint à s'aligner sur la source qui contient le moins d'informations, soit l'enquête de 1970. Le modèle ainsi construit est davantage descriptif qu'explicatif. Il répond néanmoins au but recherché d'estimer l'impact de l'érosion de l'intégration conjugale sur la progression de la part des dépressifs, et cela débarrassé des biais de la mesure intertemporelle dont il a été fait état précédemment. Un modèle pour chaque sexe est établi afin de tenir compte des éventuelles tendances contradictoires pour certaines variables indépendantes. Rappelons que l'indicateur utilisé ici tend à minimiser les niveaux de dépressivité et qu'ainsi les proportions rendent mieux compte des évolutions.

Les modifications des caractéristiques sociodémographiques de la population intervenues entre 1970 et 2002 contribuent à une augmentation du taux de la dépressivité de près de 0,5 point pour les hommes et 0,2 point pour les femmes, ce qui représente respectivement $34 \%$ et $5 \%$ de l'ensemble des augmentations de la période (Annexe 3). Ces totaux résultent du solde des contributions négatives et positives de ces caractéristiques à la prévalence de la dépression : vieillissement 
de la population, modifications des catégories socioprofessionnelles et transformations de la famille.

Les nouveaux équilibres dans la répartition et le niveau des effectifs des couples et des personnes seules ont contribué pour plus d'un cinquième à l'augmentation globale de la dépression depuis la fin des Trente Glorieuses (Tableau 6), mais celle-ci ne s'est pas réalisée uniformément. Les personnes séparées en constituent les plus forts contributeurs $(7,3 \%$ et $7,7 \%$ de la part des contributions pour les hommes et les femmes), suivies par les contributions des hommes célibataires et des monoparentes. Au total, les modifications intertemporelles des types de ménage représentent plus d'un cinquième (20,4\% pour les hommes, 22,2 \% pour les femmes) de la progression de la dépression entre 1970 et 2002 . Les situations conjugales qui ont fait progresser la dépression sont le célibat et la séparation pour les hommes. Pour les femmes, ce sont la séparation et cette forme particulière de la séparation avec enfant(s), autrement dit la monoparentalité, qui est à $85 \%$ féminine.

\section{TABLEAU 6. - Logit et décomposition logistique des risques d'être dépressif entre 1970 et 2002. Extraits de l'Annexe 3 (en \%)}

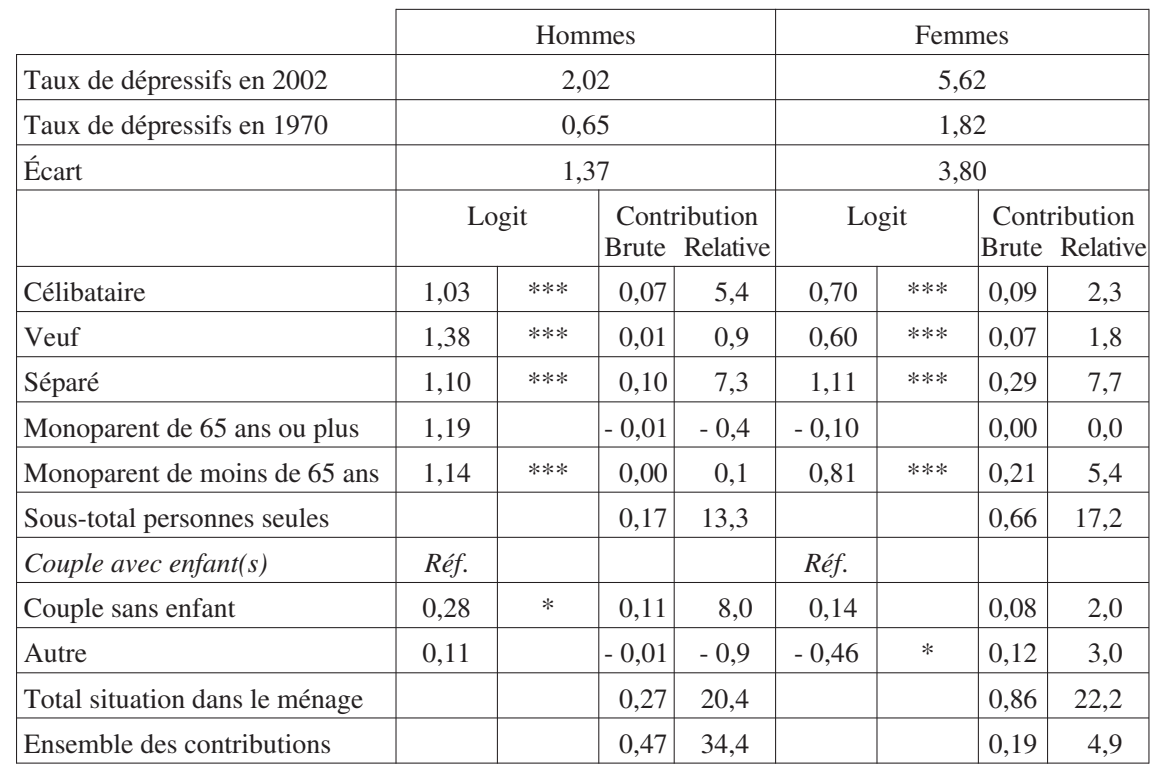

Note: $* * * \mathrm{p}<1 \%$; ** $\mathrm{p}<5 \%$; $\mathrm{p}<10 \%$.

Ce tableau est extrait du modèle complet de la décomposition statistique développé en Annexe 3 . Il présente les risques d'être dépressif (logits) calculés pour chaque sexe en empilant les deux échantillons (voir Annexe 1), puis les contributions de chaque modalité au taux de dépressifs et la part relative qui rapporte la contribution à l'écart entre les taux de dépressifs de 2002 et 1970.

Champ : Personnes de référence et conjoints.

Source : Enquêtes « Santé » 1970 et 2002.

L'aggravation de la dépressivité liée au seul affaiblissement de l'intégration conjugale est principalement due à l'accroissement du nombre de personnes sans conjoint, $13 \%$ pour les hommes et $17 \%$ pour les femmes dans la période, soit environ $15 \%$ pour les deux sexes. Si la progression de la vie sans conjoint se 
traduit bien par un alourdissement des prévalences dépressives, son impact propre demeure toutefois limité. L'évaluation de l'affaiblissement de l'intégration conjugale entre 1970 et 2002 permet de montrer que celle-ci a bien fragilisé une partie de la population, mais elle ne l'a pas fait basculer dans une " anomie conjugale généralisée ». De fait, $85 \%$ de l'augmentation de la dépression n'est pas expliquée par la progression des effectifs de solitaires, mais est redevable à d'autres causes.

\section{La dépression malade du chômage}

Quelles ont été les conséquences des transformations du salariat sur les individus, l'intensification et les modifications du management sont-elles perceptibles par le biais des catégories sociales au cours des « Trente Piteuses »? Les informations disponibles dans les enquêtes «Santé » sont limitées au statut d'activité et à la catégorie socioprofessionnelle des interviewés. Le secteur d'activité n'y figure pas, ni le degré d'autonomie, ni l'intensité du travail, ou encore le support social tiré des collègues, toutes dimensions ayant une influence sur le ressenti du travail (Karasek et Theorell, 1990). Les informations sont donc insuffisantes pour mener une investigation poussée. Mais même limitées à ces deux variables, les comparaisons temporelles au regard de la dépression sont précieuses. Les évolutions de la structure des professions et de la place des femmes ont conduit à des contributions genrées très différenciées. D'abord, la diminution du nombre de femmes au foyer a contribué à réduire la dépression de $16 \%$ entre 1970 et 2002 (Tableau 7, modalité Autre inactif). L'irruption massive des femmes sur le marché du travail salarié a objectivement contribué à leur autonomisation et à leur moindre dépendance vis-à-vis de leur conjoint masculin. Contrairement aux hommes, la salarisation des femmes a entraîné un changement de statut social par le gain d'autonomie professionnelle et familiale (Maruani, 2005). En accédant à l'emploi salarié, les femmes ont acquis à la fois des interactions de labeur, des relations de sociabilité avec les collègues davantage enrichissantes que le cantonnement au foyer, un sentiment d'émancipation surtout lorsqu'elles se comparent à leur mère (Baudelot et al., 2003), un rôle symbolique plus valorisé, et un pas vers le rééquilibrage des pouvoirs au sein des couples. Le travail est aussi l'occasion d'un épanouissement, au moins pour certaines d'entre elles.

Le développement du travail féminin a été effectivement bénéfique pour les femmes, mais ce bénéfice est inégalement stratifié. Ainsi, les contributions des femmes cadres supérieurs et moyens ont davantage réduit le niveau de la dépressivité entre 1970 et 2002 que les catégories subalternes (Tableau 7). Leurs plus grande autonomie et meilleur contrôle de leur travail sont des éléments reconnus favorisant une bonne santé mentale (Karasek et Theorell, 1990 ; Baudelot et al., 2003). Pourtant, ce bénéfice paraît davantage féminin (les coefficients des modèles logistiques en 2002 sont négatifs et significatifs pour les femmes des catégories supérieures) que masculin (les coefficients de leurs CS sont non significatifs) $\left(3^{\mathrm{e}}\right.$ et $4^{\mathrm{e}}$ colonnes de l'Annexe 2$)$. Les emplois féminins subalternes (catégorie de référence du modèle) sont aussi ceux qui sont le plus souvent à temps partiel, les moins valorisés et les plus faiblement rétribués (Maruani, 2005). C'est donc cette opposition qui est probablement captée. 
TABLEAU 7. - Logit et décomposition logistique des risques d'être dépressif entre 1970 et 2002. Extraits de l'Annexe 3

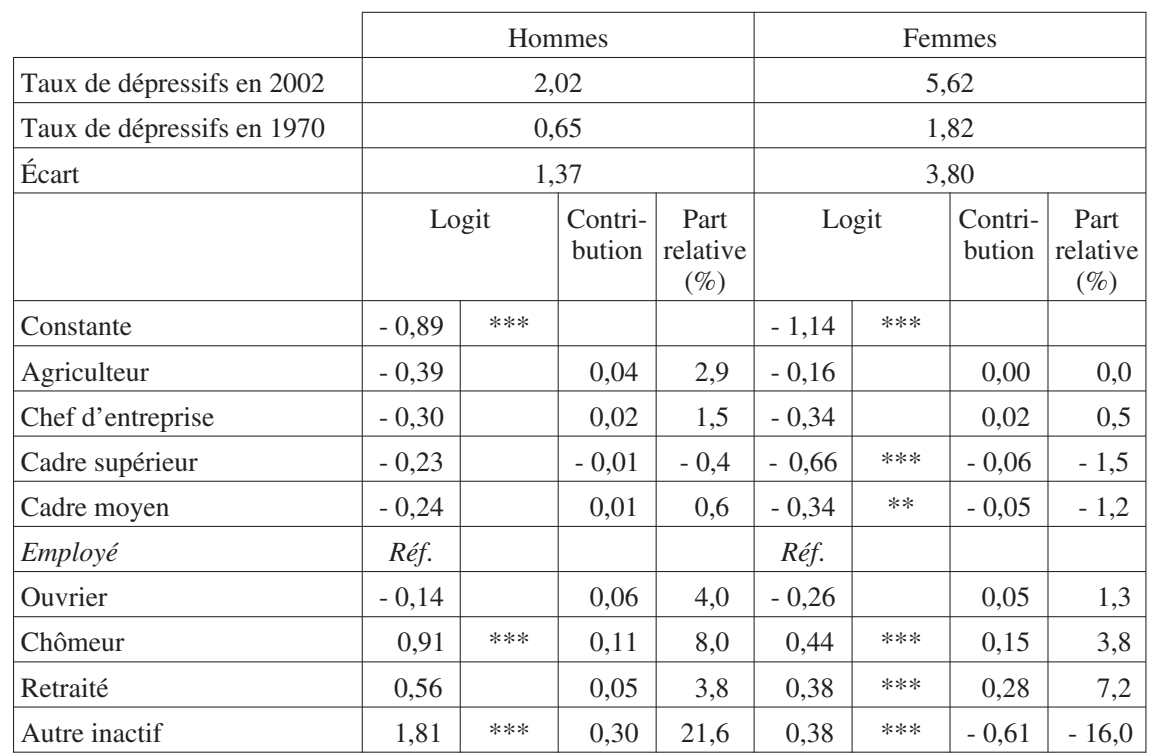

Pour les deux sexes, mais c'est d'autant plus vrai pour les hommes, être au chômage s'avère plus coûteux pour la santé mentale que de travailler. Cela actualise un résultat déjà ancien de la déstabilisation des individus lors de la perte de rôle d'actif en emploi à un moment du cycle de vie où travailler demeure la norme (Rushing, 1979). À autres variables contrôlées, les situations d'inactivité institutionnelle ou effective (retraite, chômage, autres inactivités) se soldent par un surrisque dépressif des femmes et des hommes (en dehors de la retraite pour ces derniers) (Annexe 2). Les Autres inactifs masculins, catégorie qui regroupe les étudiants, les handicapés, les personnes n'ayant jamais travaillé, font augmenter considérablement les contributions à la dépressivité. Dans une étude précédente, il a été montré que ce sont les personnes affligées d'un handicap pénalisant qui sont touchées par diverses expressions de mal-être et davantage les hommes (Cousteaux et Pan Ké Shon, 2008). En dehors de ce cas, c'est principalement l'alourdissement des effectifs de chômeurs et, dans une moindre mesure, ceux des retraités qui a fait progresser le niveau identifié de la dépression dans cette période d'aprèsTrente Glorieuses.

On voit donc que l'impact des modifications du travail sur la santé mentale des individus ne peut s'interpréter de façon univoque car, malgré un management plus agressif, le travail, comparé aux situations d'inactivité, demeure un vecteur de bonne santé mentale pour des raisons variées, changeantes et parfois incomplètes selon le statut social de l'individu (y compris son sexe) : l'affirmation d'un statut valorisant, la réalisation de soi, le gain d'autonomie et d'estime de soi, les échanges relationnels, la structuration du temps, etc. (Demazière, 1996). Cette réflexion n'est évidemment pas destinée à masquer la souffrance au travail, notamment dans les secteurs où le management se donne pour but explicite de 
« dégraisser » les effectifs des salariés les plus coûteux en les faisant «craquer » psychologiquement (Dejours et Bègue, 2009 ; Du Roy, 2009) ou les secteurs de forte intensification du travail sans autonomie pour les salariés. L'absence de significativité des coefficients liés aux catégories socioprofessionnelles des hommes par contraste avec ceux élevés et fortement significatifs des personnes seules semble indiquer que l'intensification pathologique du travail est donc loin d'avoir touché l'ensemble du salariat et tous les secteurs d'activité (Annexe 2). L'absence d'activité est d'autant plus pénalisante pour les hommes, dont le rôle social est toujours celui de breadwinner, lorsqu'elle est subie, à l'instar du chômage ou de l'invalidité. Pour les femmes, l'inactivité est un retour à la dépendance du conjoint et aux tâches dévalorisées, qui se solde par un taux de dépressivité significatif pour les trois modalités d'inactifs.

Il faut donc retenir, premièrement, que les situations délétères masculines proviennent principalement des formes de vie solitaire. Elles sont plus nocives que la position sur l'échelle des catégories sociales mais de niveau quasi similaire avec le chômage ; deuxièmement, en ce qui concerne les femmes, les catégories sociales modestes sont davantage pénalisées. Troisièmement, le chômage se montre davantage pathologique que le travail, pour les hommes ou les femmes. Pour ces dernières, même la retraite est plus dépressive que l'activité salariée. À cette étape, nous serions donc amenés à penser que la précarisation des statuts du travail salarié entraînant des épisodes plus ou moins longs et répétés de chômage et le déficit d'intégration conjugale sont les éléments principaux qui ont érodé l'intégration des individus dans cette période. Nous pouvons reprendre mot pour mot ce que Bastide relevait en 1965 : « Nous retrouvons ici le problème posé par A. Comte, et que l'on ne peut ignorer : la corrélation entre les faits de désorganisation personnelle et de désorganisation sociale ; les faits montrent, d'une façon impressionnante, que les désordres mentaux sont plus nombreux dans les secteurs désintégrés que dans les secteurs intégrés d’une population donnée. » (p.93).

\section{La dépression contrariée par le suicide}

Il reste que l'on ne sait dire, depuis le milieu du $\mathrm{XX}^{\mathrm{e}}$ siècle, si la transition des régimes réglementaires s'est réellement accompagnée d'une augmentation des tensions mentales ou si les déplacements des tensions d'un secteur à l'autre de la société ou d'une pathologie à une autre, suivant parfois les seuls soubresauts nosographiques, les a rendues seulement plus visibles. De leur côté, la meilleure dicibilité des maladies psychiques et l'amélioration de leur dépistage entachent les comparaisons intertemporelles de leurs niveaux du doute irréductible du biais, sans que l'on puisse en évaluer son importance. À l'inverse de la dépression, le recueil des certificats de décès pour cause de suicide échappe à ce type de biais. Le suicide paraît un indicateur plus stable que la dépression et susceptible de fournir un contrepoint utile. Il n'est pas sans reproche, car lui aussi est orienté par le genre (en France les suicides masculins en 2006 sont trois fois plus nombreux que les suicides féminins), les données accessibles ne permettent pas de réaliser des études micro-économétriques et il est fortement spécifique par sa radicalité. La sous-déclaration, autre reproche souvent avancé, était jusqu'à présent estimée grossièrement à $20 \%$, mais une étude plus récente, faisant appel à un protocole adapté, l'évalue à près de $10 \%$, sans écarts sexués réellement significatifs (Aouba et al., 2011). Mais, 
surtout, la sous-déclaration doit être stable au cours des trois ou quatre dernières décennies à cause du contrôle policier sur les morts violentes et de l'établissement de la cause du décès par un médecin. En ce sens, les comparaisons temporelles ne devaient pas s'en trouver pénalisées ou alors très marginalement.

Les évolutions du suicide au cours du temps instillent une complexité inattendue dans la lecture trop linéaire des liens entre modification du régime normatif, individualisation et augmentation des tensions. Ainsi, l'évolution temporelle de la mortalité par suicide, cette expression radicale du mal-être des individus, illustre une mutation qui semble davantage être corrélée avec le chômage (Figure 2). Au cours des Trente Glorieuses, le niveau du suicide masculin est demeuré faible et sans variations significatives, à l'instar d'un taux de chômage extrêmement réduit et stable. Cette période passée, les taux du suicide et du chômage augmentent vigoureusement jusqu'au milieu des années 1980 pour retomber ensuite jusqu'en fin de période d'observation, avec un soubresaut au milieu des années 1990. Quant aux femmes, leur mortalité suicidaire progresse continûment de 1945 à 1986, puis retombe (avec le même soubresaut mais de bien moindre amplitude) pour se stabiliser, contrairement aux hommes, au niveau haut d'avant la Seconde Guerre mondiale. Régulièrement, les observateurs sont tentés de relier mécaniquement ces évolutions aux perturbations économiques et notamment la montée du chômage, dont le profil de la courbe est proche de celui des suicides masculins (Figure 2). Ce qui a fait dire au psychiatre Michel Debout dans une interview au journal Le Monde que « la corrélation entre chômage et suicide est pourtant indéniable » et qu'il fallait s'attendre à une prochaine augmentation (Le Monde, 08-02-2012).

\section{FIGURE 2. - Évolution des taux bruts de mortalité par suicide de 1925 à 2006}

Taux de suicides pour 100000

Taux de chômage en \%

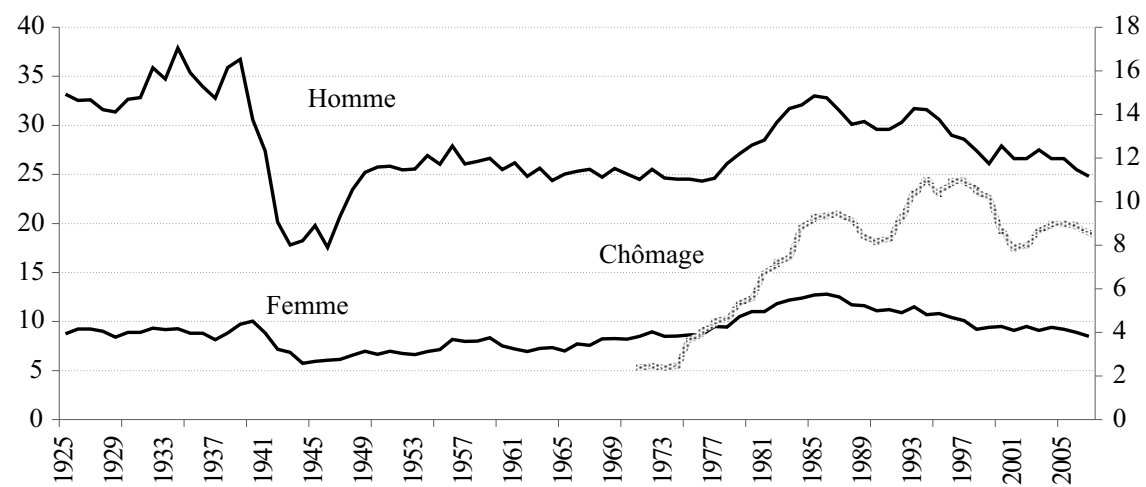

Note : Les données ne sont disponibles qu'à partir de 1975. Le profil des courbes du chômage masculin et féminin est semblable, seul le niveau varie, il est plus élevé pour les femmes.

Champ : Ensemble de la population de France.

Source suicides : Ined, 1925 à 1999 (Vallin et Meslé, 1998), CépiDc au-delà de 1999.

Source chômage : Insee, http://www.insee.fr/fr/themes/detail.asp?reg_id=0\&ref_id=ir-martra10\&page =irweb/martra10/dd/martra10_paq3.htm. Graphique des auteurs.

Chauvel a montré que les profils des courbes du suicide masculin et du chômage dessinent deux pics surgissant aux environs de 1985 et aux environs de 
1995. Ces pics du suicide coïncident avec les années noires de l'économie, quand au contraire 1985-1990 voit une période de relative embellie économique et une forte baisse des taux de suicide. Il précise plus loin : «La tentation est forte de s'arrêter à l'idée simpliste d'un suicide "économique", et de réduire les causes du suicide au seul chômage, ou aux difficultés économiques, autrement dit aux aspects les plus matériels de la "crise". » (1998, p. 3). Pour conforter son analyse, il s'appuie sur le texte de Halbwachs : «Ce n'est pas que la misère des ouvriers qui chôment, les banqueroutes, les faillites et les ruines soient la cause immédiate de beaucoup de suicides. Mais un sentiment obscur d'oppression pèse sur toutes les âmes, parce qu'il y a moins d'activité générale, que les hommes participent moins à une vie économique qui les dépasse, et que leur attention n'étant plus tournée vers le dehors se porte davantage non seulement sur leur détresse ou leur médiocrité matérielle, mais sur tous les motifs individuels qu'ils peuvent avoir de désirer la mort. » ([1930] 2002, p. 394). Peut-être faut-il penser plus simplement qu'il n'y a pas un lien direct entre chômage et suicide car l'effet du chômage est médiatisé par certains éléments dont le support social plus ou moins efficace des individus. De plus, les aspects nocifs du chômage et des crises économiques se diffusent aussi parmi les proches à cause des tensions interpersonnelles induites ou comme un élément dépressif s'ajoutant à d'autres sur le mode de " la goutte d'eau qui fait déborder le vase ». Le chômage crée aussi un nouveau rapport de force entre employeurs et employés, susceptible de généraliser les tensions à l'ensemble des salariés, la baisse d'activité ralentit l'activité des commerçants, etc.

L'examen des taux de suicide par tranches d'âge décennales et par genre entre 1979 et 2009 montre que le suicide n'a pas touché que les actifs, mais bien l'ensemble des individus, cela quels que soient leurs âges. Les catégories qui semblent avoir été les plus drastiquement affectées par la crise économique et les pics du chômage sont les personnes dont l'âge les éloigne de l'activité professionnelle ${ }^{11}$. C'est particulièrement net pour les tranches d'âge des hommes au-delà de 55 ans et pour la quasi-totalité des tranches d'âge des femmes (Figure 3). Les facteurs agissant sur le suicide sont multidimensionnels et réduire l'explication de l'alourdissement ponctuel de la suicidité à un seul d'entre eux risque fortement d'entraîner des conclusions erronées. Plusieurs remarques modératrices peuvent être avancées. Premièrement, les cohortes nées en 1890, 1900, 1910 sont davantage suicidaires que les suivantes. Elles avaient, en 1985, 95 ans, 85 ans et 75 ans (Anguis, Cases et Surault, 2002), ce qui pourrait expliquer, au moins partiellement, la sursuicidité des personnes de plus de 75 ans par rapport aux décennies antérieures et postérieures. Deuxièmement, il y a bien eu un effet de période qui a touché tous les âges pour les deux sexes, en dehors des femmes de 15-24 ans, dont le taux de suicide est habituellement très bas. L'amplitude des taux de suicidité selon les âges tend à écraser visuellement les taux les plus modérés (Figure 3).

Le calcul d'un ratio simple des taux de suicide pallie ce problème. Il suffit de rapporter les taux de suicidité pendant la période de forte accentuation $\mathrm{du}$ chômage à ceux d'une année de référence où le chômage est " ordinaire » ou contenu. Ainsi, les taux de suicidité des années 1985 et 1986 sont additionnés afin de disposer d'effectifs suffisants, puis ils sont divisés par deux pour lisser les

11. Incidemment, on soulignera que le taux de suicide des personnes âgées, pourtant spectaculairement élevé, suscite peu l'attention commune, révélant ainsi une sorte d'échelle d'intensité de la compassion selon les âges. 
effets sur ces deux années pivots et, enfin, ils sont rapportés aux taux de 1979, année choisie conventionnellement pour la modération de ses taux. Les résultats montrent que les écarts des ratios sont faibles entre chaque tranche d'âge et entre sexes (Tableau 8). Seuls les 45 à 54 ans pour les femmes ont un taux de suicide 1,4 fois supérieur à 1979 et les hommes de 75 à 84 ans affichent un ratio de près de 1,3 fois plus élevé. Troisièmement, tous les taux de suicide masculin sont en 2009 inférieurs à ceux observés avant le début de la crise (prise ici en 1979 à cause de la disponibilité des données) pour les mêmes âges, en dehors des 35-44 ans. De façon similaire, à tous les âges, les taux de suicide féminin baissent en 2009 par rapport à 1979 (en dehors des 45 à 54 ans, dont les taux augmentent de 0,5 pour 100000$)$.

\section{FIGURE 3. - Évolution des taux bruts de mortalité par suicide de 1979 à 2009}

\section{Taux pour 100000}
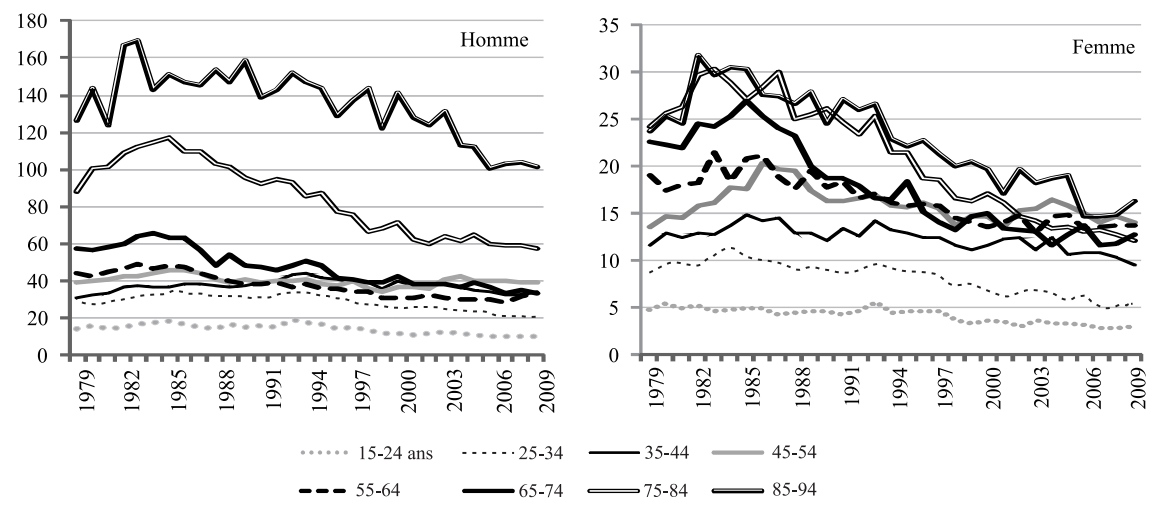

Champ : Ensemble de la population de France.

Source : CépiDc. Graphique des auteurs.

TABLEAU 8. - Ratio des taux de suicide 1985 et 1986 sur 1979

\begin{tabular}{|c|c|c|}
\cline { 2 - 3 } \multicolumn{1}{c|}{} & Homme & Femme \\
\hline $15-24$ ans & 1,18 & 1,02 \\
\hline $25-34$ ans & 1,22 & 1,17 \\
\hline $35-44$ ans & 1,21 & 1,25 \\
\hline $45-54$ ans & 1,18 & $\mathbf{1 , 4 0}$ \\
\hline $55-64$ ans & 1,09 & 1,10 \\
\hline $65-74$ ans & 1,10 & 1,15 \\
\hline $75-84$ ans & $\mathbf{1 , 2 8}$ & 1,15 \\
\hline $85-94$ ans & 1,18 & 1,22 \\
\hline
\end{tabular}

Les évolutions des taux de suicide au cours du temps suggèrent que :

1) Il y a bien eu des aggravations des tensions depuis les années 1970, sensibles dans les années 1980 et 1990. Conséquemment, on peut en déduire que la montée de la dépression, très corrélée au suicide (Sher, Oquendo et Mann, 2001 ; Hawton 
et Heeringen, 2009), n'est pas uniquement due à l'amélioration du dépistage et de la dicibilité mais qu'elle a aussi augmenté ;

2) Le pic du chômage en 1985 s'est bien accompagné d'une montée des suicides quel que soit l'âge (en dehors des moins de 25 ans) et pour les deux sexes ;

3) Les niveaux du suicide des hommes et des femmes sont très majoritairement inférieurs en 2009 aux niveaux pourtant déjà relativement bas de 1979 ;

4) Les tensions dues au nouveau management ne sont pas perceptibles par les courbes du suicide;

5) Les tensions dues à la montée progressive et régulière des effectifs de personnes seules après la période 1965-1975 devraient se traduire par une progression continue linéaire et non pas heurtée. En outre, le niveau n'a pas de raison de baisser brutalement (Figure 2).

Il est remarquable que les taux masculins du suicide, au début des années 2000, soient revenus au niveau des années 1970, sans pour autant que la conjoncture économique soit aussi souriante. À l'inverse, le management est devenu plus agressif et les parts de personnes seules ont progressé. Ce qui revient à dire que les taux de suicide actuels devraient être encore inférieurs aux niveaux bas de 1970, si ces facteurs aggravants étaient contrôlés statistiquement dans la comparaison de la suicidité à ces deux dates. On peut s'étonner de ce paradoxe apparent. Cela proviendrait-il du développement de l'autocontrôle de la violence, et donc de la violence sur soi, au cours du processus continu de civilisation (Elias, [1939] 1990) ? Mais ce type d'évolution sociétale possède une inertie certaine et l'on s'attend à ce que les mouvements de fond soient lents, progressifs et imperceptibles « à l'œil nu », à l'inverse des variations vigoureuses du suicide observées dans les dernières décennies. L'hypothèse alternative partirait des conditions socioéconomiques « anormalement » troublées à la sortie des Trente Glorieuses et qui, au fil du temps, ont muté en état ordinaire. De fait, les générations d'après 1973 n'ont connu que cet état de l'emploi et de l'économie, ce qui leur confère une banalité certaine.

\section{Discussion et conclusion}

Les niveaux identiques de la dépressivité entre le célibat et le veuvage-état révèlent la part commune à ces deux situations de vie : le manque commun d'intégration conjugale (Tableau 5). Ce n'est pas le moindre des résultats de constater que, à autres variables sociodémographiques contrôlées, veufs-état et célibataires réagissent avec un même niveau de dépressivité au manque d'intégration conjugale, autrement dit à l'absence structurelle de support social venant du conjoint. La distinction du choc de l'événement du veuvage et de l'état ordinaire de veuf a permis d'isoler dans nos résultats deux éléments de la régulation au cours de ce choc : la dérégulation par la déstructuration ponctuelle du quotidien et l'ébranlement émotionnel de la perte du conjoint (perte de soutien par la séparation ou le décès). Jusqu'à présent nous étions globalement demeurés sur le modèle durkheimien du dysfonctionnement social et du dysfonctionnement du lien conjugal, notamment par le divorce, aboutissant au suicide. Avec la banalisation de la vie sans conjoint, il est devenu impossible de considérer le célibat, la séparation et a fortiori le 
veuvage comme des dysfonctionnements sociaux, et il faut plus simplement les observer comme de nouvelles façons de vivre les rapports aux autres. Halbwachs remarquait avec finesse : «Mais si les suicides, au contraire, augmentent surtout parce que la vie sociale se complique, et que les événements singuliers qui exposent au désespoir s'y multiplient, ils sont toujours un mal, mais peut-être un mal relatif. Il y a en effet une complication nécessaire qui est la condition d'une vie sociale plus riche et plus intense. » ([1930] 2002, p. 12).

Les constats répétés de sociétés plus complexes et pathogènes (Tocqueville, [1840] 1951 ; Janet, 1929 ; Halbwachs, [1930] 2002 ; Bastide, 1965 ; Ehrenberg, 1998) conduisent à s'interroger sinon sur leur validité du moins sur leur portée. Dès lors que le constat initial est effectivement crédité, il faudrait alors s'attendre à la dégradation continue de la santé mentale des individus et conséquemment à l'alourdissement de la prévalence des diverses expressions de mal-être. Or, ce n'est pas ce qui est observé à partir du suicide. Les chocs économiques et du chômage de masse qui ont profondément ébranlé la société française à la sortie des Trente Glorieuses se sont bien transmis à l'ensemble des individus, même aux retraités moins directement impliqués dans le fonctionnement productif et les moins touchés matériellement par ses conséquences (Figure 3, Tableau 8). Les constats du choc du veuvage versus son état convergent avec ceux de la montée vigoureuse du chômage après le premier choc pétrolier, puis de sa stabilisation. Au choc du veuvage succède, après une période d'absorption de ce choc, l'état ordinaire de veuf. Aux chocs du chômage de masse et des crises économiques se substituerait progressivement, et au cours du temps qui passe, un nouvel état qui ne serait rien d'autre que la redéfinition de l'état antérieur en état courant, autrement dit en état ordinaire. Les chocs sociaux en tant qu'événements ont laissé place à une situation sociale plus instable mais qui s'érige progressivement en normalité, cela d'autant plus pour ceux n'ayant pas ou peu vécu la situation antérieure des Trente Glorieuses. Les repères des individus sont modifiés, transmutant l'ancienne anormalité en nouvelle normalité semblant simultanément neutraliser, au moins en partie, la nocivité d'hier. Ce qui explique que les constats répétés au cours du temps d'une société toujours plus pathogène n'aient pas débouché sur une forme de société cannibale, dévorant avec un appétit toujours plus féroce ses propres membres.

Le rapport dialectique du stress et de l' «adaptation » à mesure du temps qui passe ouvre un large champ d'investigations sociologiques. Jusqu'à quel point de tension l'adaptation est-elle possible ? Peut-on s'adapter à chaque type de choc ? Les individus sont-ils également adaptables ou existe-t-il ici aussi une stratification indiquant des disparités de résilience ou de coping (Pearlin et Schooler, 1978) ? Quels sont les ressorts de l'adaptabilité ? La plasticité des agents, dont on ne connaît ni la portée ni les mécanismes, aux nouvelles configurations sociales et intimes, leur seuil de résistance aux tensions mentales constituent des pistes de recherche peu aisées à emprunter, mais potentiellement riches dans la compréhension du trio sociétés, tensions et individus. Pour modérer ces réflexions, il faut rappeler que la période d'adaptation que nous avons observée court sur plusieurs décennies et que celle-ci a été particulièrement suicidogène (Graphiques 2 et 3, Tableau 8). Ensuite, si au regard du suicide le chômage paraît moins nocif que par le passé, la dépression montre toujours qu'il érode davantage la résistance des chômeurs que celle des actifs en emploi (Tableau 7). Enfin, plus prosaïquement, 
les personnes qui ont davantage connu l'état antérieur des Trente Glorieuses sont celles aussi qui quittent ou ont déjà quitté le marché du travail.

L'étude conjointe de la dépression et du suicide permet de dépasser la tentation de l'égalité stricte : sociologie des tensions mentales égale sociologie de l'inscription dans l'intimité des esprits des dysfonctionnements sociaux et de la dérégulation. De fait, certaines pathologies mentales surgissent à cause de situations sociales toxiques, par exemple, le nouveau management (Boltanski et Chiapello, 1999), mais il ne faudrait pas en conclure trop simplement que toutes les tensions sont à mettre sur le compte de dysfonctionnements qu'il suffirait d'éliminer pour parvenir soudain à un monde sans tensions. L'énoncer ainsi montre déjà la naïveté de cet improbable attendu. Aneshensel, résumant la pensée de Merton (1938), avance que l' " ordre social permet le fonctionnement émotionnel normal mais produit aussi des circonstances où le désordre émotionnel constitue une réponse normale ou prévisible. On peut voir la présence de stress social, donc, comme une conséquence inévitable de l'organisation sociale » (Aneshensel, 1992, p. 33 ; c'est nous qui soulignons). Merton soulignait les contradictions génératrices de tensions entre de fortes normes culturelles promouvant la réussite pécuniaire et une structure sociale n'autorisant qu'une partie de la population à y accéder. Aneshensel va plus loin en soutenant que «les impératifs associés à la maintenance du système social créent inévitablement des tensions entre l'individu et la collectivité. Ces sources systémiques de tension peuvent être changées d'un emplacement du système à un autre, mais ne peuvent pas être éliminées entièrement. Ainsi, des circonstances de vie stressantes et leurs conséquences émotionnelles peuvent être et sont souvent éprouvées par des gens parfaitement ordinaires intégrés dans les structures normatives de la société » (ibid., p. 33). De fait, les écarts de prévalence des tensions mentales entre hommes et femmes, la sélectivité de celles-ci en fonction du genre sont aussi issus d'un fonctionnement «normal » d'une société où la régulation des individus varie selon leur socialisation et leurs places assignées (par le genre, l'âge, la classe sociale, l'ethnicité, etc.) et non pas seulement à cause de dysfonctionnements sociaux. Prenant un pas de recul et plaçant sa réflexion au niveau des valeurs de chaque société, Pierre-Henri Castel affirme qu' « il n'existe peut-être pas de véritable vie sociale dans laquelle on ne puisse pas devenir malade, et pourquoi pas psychiquement malade, et jusqu'à en mourir, de ce qui donne sens à l'existence collective : de ses valeurs incarnées » (2013, p. 9). Le glissement du régime de régulation de l'individu de l'obéissance à l'autonomie fait qu' "il s'accompagne nécessairement d'une modification dans la manière de subir » (Ehrenberg, 2010, p. 348). Plus globalement, le processus de civilisation s'accompagne de "changements qui affectent les normes sociales concernant les comportements et les sentiments humains, et par conséquent les maladies qui leur sont liées»(Elias, [1988] 2010, p. 115). La sociologie des tensions mentales dévoile non seulement ce que tout système de contention et d'autorisation contient de pathologique, mais, plus simplement, ce que tout système de régulation (de valeurs et de normes) produit nécessairement comme effets toxiques. Les sociétés, même dans leur logique harmonieuse de fonctionnement, produisent des tensions et des pathologies mentales qui leurs sont spécifiques.

Dans une dynamique sociétale où les unions sont plus rares, moins contraignantes et plus instables, nous sommes amenés à penser que l'accroissement probable du groupe des personnes sans conjoint continuera à nourrir les effectifs 
des diverses formes de mal-être et notamment de la dépression. L'intégration conjugale s'est effritée, particulièrement depuis la seconde transition démographique, et en trente ans elle a contribué à l'augmentation de $15 \%$ de la prévalence dépressive. Ce n'est pas le moindre des paradoxes que la libération du couple, autrefois perçu comme réactionnaire, et l'extension de l'autonomie des individus se traduisent par des pathologies révélant leur fragilisation. L'alourdissement des effectifs de dépressifs consécutif à l'affaiblissement de l'intégration conjugale viendra, comme au cours de ces trente dernières années, des séparés et des monoparents. Mais il faut se garder des interprétations univoques et des jugements intempestifs. L'entrée dans la vie adulte sans conjoint correspond aussi à l'apprentissage des rapports aux autres, contribuant à la construction de soi et à l'affinement des choix et de la personnalité des individus. En outre, les unions sont loin d'être toutes harmonieuses. Les conflits entre conjoints se traduisent par des problèmes d'alcoolisme chez les hommes et de dépression chez les femmes (Horwitz et White, 1991). La séparation, y compris pour les monoparents et au-delà des difficultés matérielles, peut se révéler moins nocive que la mésentente conjugale (Simon, 2002). La déliaison conjugale, l'individualisation du sujet se paient donc par des pathologies de la modernité ou, de façon moins mélodramatique, il faut simplement constater que les diverses configurations sociétales s'accompagnent de leurs tensions mentales singulières.

Jean-Louis PAN KÉ SHON CREST-LSQ
60, rue Étienne Dolet
92240 Malakoff
jean-louis.pan-ke-shon@ined.fr

Géraldine DuTHÉ

Institut national d'études démographiques 133, boulevard Davout 75980 Paris cedex 20 geraldine.duthe@ined.fr

\section{ANNEXE 1. - Décomposition statistique de Blinder-Oaxaca appliquée aux logits}

\footnotetext{
La méthode proposée par Fairlie (2005) suit plusieurs étapes. Une indicatrice identifiant l'enquête de 2002 est introduite afin de capter les effets spécifiques de cette année. Le modèle logistique est estimé sur les données empilées des enquêtes «Santé » de 1970 et 2002. Les effectifs des deux enquêtes sont égalisés sur le plus petit échantillon. Les effectifs de l'enquête avec l'échantillon initialement plus important sont tirés aléatoirement un certain nombre de fois (ici 25) afin d'éviter les artefacts dus à la déformation de l'échantillon venant de la troncature. La chaîne de calculs est réalisée à chaque itération. Les coefficients des paramètres estimés grâce au modèle logistique sont ceux provenant de ces deux enquêtes empilées. Ils correspondent à l'impact de chaque modalité des variables du modèle sur la dépression. Les contributions de chaque variable indépendante à l'écart entre 2002 et 1970 sont ensuite calculées en fonction de ces coefficients communs et des variables indépendantes de chaque enquête. Les calculs des probabilités individuelles d'être dépressif varient alors selon la seule composition de la population à chaque date
} 
d'enquête. Les résultats sont ainsi débarrassés des effets de période. La formalisation de la décomposition d'une équation $Y=F(X \widehat{\beta})$ s'écrit de la façon suivante (Fairlie, 2005) :

$$
\bar{Y}^{70}-\bar{Y}^{02}=\left[\sum_{i=1}^{N^{70}} \frac{F\left(X_{i}^{70} \widehat{\beta}_{i}^{70}\right)}{N^{70}}-\sum_{i=1}^{N^{02}} \frac{F\left(X_{i}^{02} \widehat{\beta}_{i}^{70}\right)}{N^{02}}\right]+\left[\sum_{i=1}^{N^{02}} \frac{F\left(X_{i}^{02} \widehat{\beta}_{i}^{70}\right)}{N^{02}}\right]-\left[\sum_{i=1}^{N^{02}} \frac{F\left(X_{i}^{02} \widehat{\beta}_{i}^{02}\right)}{N^{02}}\right]
$$

Où :

$F$ représente la fonction cumulative de la distribution logistique ;

$\bar{Y}^{70}-\bar{Y}^{02}$ représente l'écart du risque d'être dépressif entre 1970 et 2002 ;

$N^{70}$ et $N^{02}$ représentent les effectifs de 1970 et 2002 ;

$X^{70}$ et $X^{02}$ représentent des vecteurs de variables indépendantes en 1970 et 2002 ;

$\hat{\beta}_{i}^{70}$ et $\widehat{\beta}_{i}^{02}$ représentent des vecteurs de coefficients estimés pour les variables indépendantes en 1970 et 2002 .

Le premier terme entre crochets représente la part de l'écart entre deux dates qui est due aux différences des effectifs dans les distributions de $X$ (structure) et le second la partie due aux différences des processus déterminant les niveaux d' $Y$. Ce dernier membre capte également les effets des variables inobservées et inobservables.

Les contributions de chaque variable indépendante à l'écart entre 2002 et 1970 sont ensuite calculées. L'identification de la contribution des différences de variables spécifiques à l'écart de la dépression entre deux dates n'est pas directe. La contribution de chaque variable à l'écart de la dépressivité entre deux dates est égale au changement de la probabilité prédite moyenne du remplacement de la distribution de 1970 avec la distribution de 2002 de cette variable tout en maintenant les distributions des autres variables constantes. Les contributions de chaque variable aux variations de la dépression entre les périodes sont donc estimées successivement et de façon glissante. Ainsi, la contribution de $X_{l}$ à l'écart entre les deux dates s'exprime par:

$$
\frac{1}{N^{70}} \sum_{i=1}^{N^{70}} F\left(\widehat{\alpha}^{*}+X_{1 i}^{02} \widehat{\beta}_{1}^{*}+X_{2 i}^{02} \widehat{\beta}_{2}^{*}\right)-F\left(\widehat{\alpha}^{*}+X_{1 i}^{70} \widehat{\beta}_{1}^{*}+X_{2 i}^{02} \widehat{\beta}_{2}^{*}\right)
$$

Puis la contribution de $X_{2}$ à l'écart s'obtient par :

$$
\frac{1}{N^{70}} \sum_{i=1}^{N^{70}} F\left(\widehat{\alpha}^{*}+X_{1 i}^{70} \widehat{\beta}_{1}^{*}+X_{2 i}^{02} \widehat{\beta}_{2}^{*}\right)-F\left(\widehat{\alpha}^{*}+X_{1 i}^{70} \widehat{\beta}_{1}^{*}+X_{2 i}^{70} \widehat{\beta}_{2}^{*}\right)
$$

Où :

$N^{70}$ et $N^{02}$ représentent les effectifs de 1970 et 2002 ;

$\widehat{a}^{*}$ correspond à la constante ;

$X_{1 i}^{70}$ et $X_{1 i}^{02}$ représentent la même variable indépendante en 1970 et 2002 pour l'individu $i$;

$\widehat{\beta}_{1}$ et $\widehat{\beta}_{2}$ représentent les coefficients estimés sur les échantillons empilés de 1970 et de 2002 pour la première et la seconde variables indépendantes. 
ANNEXE 2. - Risque d'être dépressif, modèles logistiques

\begin{tabular}{|c|c|c|c|c|c|c|c|c|}
\hline & \multicolumn{4}{|c|}{ Enquête de 1970} & \multicolumn{4}{|c|}{ Enquête de 2002} \\
\hline Constante & \multicolumn{4}{|c|}{$-4,3 * * *$} & \multicolumn{4}{|c|}{$-4,3^{* * * *}$} \\
\hline Sexe & \multicolumn{4}{|c|}{0,3} & \multicolumn{4}{|c|}{$1,5 * * *$} \\
\hline & \multicolumn{2}{|c|}{ Hommes } & \multicolumn{2}{|c|}{ Femmes } & \multicolumn{2}{|c|}{ Hommes } & \multicolumn{2}{|c|}{ Femmes } \\
\hline Célibataire & 1,2 & $* *$ & 0,2 & & 1,1 & $* * *$ & 0,8 & $* * *$ \\
\hline Veuf & 1,1 & & $-0,1$ & & 1,5 & $* * *$ & 0,7 & $* * *$ \\
\hline Séparé & $-12,2$ & & 1,0 & & 1,2 & $* * *$ & 1,1 & $* * *$ \\
\hline Monoparent de 65 ans ou plus & $-10,9$ & & 0,1 & & 1,6 & & $-0,1$ & \\
\hline Monoparent de moins de 65 ans & $-12,3$ & & 1,0 & $* * *$ & 1,3 & $* * *$ & 0,8 & $* * *$ \\
\hline Couple avec enfant(s) & Réf. & & Réf. & & Réf. & & Réf. & \\
\hline Couple sans enfant & 0,0 & & 0,1 & & 0,4 & $*$ & 0,2 & \\
\hline Autre & $-0,2$ & & $-0,6$ & & 0,2 & & $-0,4$ & \\
\hline 18 à 29 ans & $-2,2$ & $* *$ & $-0,5$ & & $-1,4$ & $* * *$ & $-1,2$ & $* * *$ \\
\hline 30 à 39 ans & $-0,1$ & & 0,1 & & $-0,1$ & & $-0,5$ & $* * *$ \\
\hline 40 à 49 ans & Réf. & & Réf. & & Réf. & & Réf. & \\
\hline 50 à 59 ans & $-0,2$ & & $-0,1$ & & $-0,1$ & & 0,0 & \\
\hline 60 à 69 ans & $-2,4$ & $* * *$ & $-0,7$ & $*$ & $-0,5$ & & $-0,3$ & $*$ \\
\hline 70 ans et plus & $-3,1$ & $* *$ & $-1,1$ & $* *$ & $-1,0$ & $* *$ & $-0,7$ & $* * *$ \\
\hline Agriculteur & 0,0 & & 0,8 & & $-0,8$ & & $-0,4$ & \\
\hline Chef d'entreprise & $-0,8$ & & 0,6 & & $-0,1$ & & $-0,7$ & $*$ \\
\hline Cadre supérieur & $-0,9$ & & $-0,8$ & & $-0,1$ & & $-0,7$ & $* * *$ \\
\hline Cadre moyen & $-0,4$ & & 0,1 & & $-0,2$ & & $-0,4$ & $* *$ \\
\hline Employé & Réf. & & Réf. & & Réf. & & Réf. & \\
\hline Ouvrier & $-0,4$ & & $-0,6$ & & 0,0 & & $-0,2$ & \\
\hline Chômeur & $-12,3$ & & 0,5 & & 1,0 & $* * *$ & 0,5 & $* * *$ \\
\hline Retraité & 1,0 & & 0,4 & & 0,4 & & 0,3 & $*$ \\
\hline Autre inactif & 1,6 & $* *$ & 0,2 & & 1,9 & $* * *$ & 0,4 & $* * *$ \\
\hline Nul/Non déclaré & $-0,4$ & & 0,0 & & 0,2 & & $-0,2$ & $* *$ \\
\hline$C E P, B E P C, C A P, B E P$ & Réf. & & Réf. & & Réf. & & Réf. & \\
\hline Niveau baccalauréat ou supérieur & 0,4 & & 0,5 & & $-0,1$ & & $-0,1$ & \\
\hline
\end{tabular}

Note: $* * * \mathrm{p}<1 \% ; * * \mathrm{p}<5 \% ; * \mathrm{p}<10 \%$.

Un modèle de régression logistique par enquête (variables croisées avec le sexe).

Champ : Personnes de référence et conjoint-e-s âgé-e-s d'au moins 18 ans.

Source : Enquêtes « Santé » 1970 et 2002. 
ANNEXE 3. - Risque d'être dépressif, modèles et décompositions logistiques, 1970 et 2002

\begin{tabular}{|c|c|c|c|c|c|c|c|c|}
\hline & \multirow{2}{*}{\multicolumn{4}{|c|}{ Hommes }} & \multirow{2}{*}{\multicolumn{4}{|c|}{ Femmes }} \\
\hline & & & & & & & & \\
\hline Taux de dépressifs en 2002 & \multicolumn{4}{|c|}{2,02} & \multicolumn{4}{|c|}{5,62} \\
\hline Taux de dépressifs en 1970 & \multicolumn{4}{|c|}{0,65} & \multicolumn{4}{|c|}{1,82} \\
\hline \multirow[t]{3}{*}{ Écart } & \multicolumn{4}{|c|}{1,37} & \multicolumn{4}{|c|}{3,80} \\
\hline & \multirow{2}{*}{\multicolumn{2}{|c|}{ Logit }} & \multicolumn{2}{|c|}{ Contribution } & \multirow{2}{*}{\multicolumn{2}{|c|}{ Logit }} & \multicolumn{2}{|c|}{ Contribution } \\
\hline & & & \multirow[t]{2}{*}{ Nette } & \multirow[t]{2}{*}{$\begin{array}{c}\text { Relative } \\
(\%)\end{array}$} & & & \multirow[t]{2}{*}{ Nette } & \multirow[t]{2}{*}{$\begin{array}{l}\text { Relative } \\
(\%)\end{array}$} \\
\hline Constante & $-0,89$ & $* * *$ & & & $-1,14$ & $* * *$ & & \\
\hline Célibataire & 1,03 & $* * *$ & 0,07 & 5,4 & 0,70 & $* * *$ & 0,09 & 2,3 \\
\hline Veuf & 1,38 & $* * *$ & 0,01 & 0,9 & 0,60 & $* * *$ & 0,07 & 1,8 \\
\hline Séparé & 1,10 & $* * *$ & 0,10 & 7,3 & 1,11 & $* * *$ & 0,29 & 7,7 \\
\hline Monoparent de 65 ans ou plus & 1,19 & & $-0,01$ & $-0,4$ & $-0,10$ & & 0,00 & 0,0 \\
\hline Monoparent de moins de 65 ans & 1,14 & $* * *$ & 0,00 & 0,1 & 0,81 & $* * *$ & 0,21 & 5,4 \\
\hline Couple avec enfant(s) & Réf. & & & & Réf. & & & \\
\hline Couple sans enfant & 0,28 & $*$ & 0,11 & 8,0 & 0,14 & & 0,08 & 2,0 \\
\hline Autre & 0,11 & & $-0,01$ & $-0,9$ & $-0,46$ & $*$ & 0,12 & 3,0 \\
\hline 18 à 29 ans & $-1,51$ & $* * *$ & $-0,17$ & $-12,4$ & $-1,05$ & $* * *$ & $-0,17$ & $-4,6$ \\
\hline 30 à 39 ans & 0,12 & & 0,00 & $-0,3$ & $-0,38$ & $* * *$ & 0,00 & 0,0 \\
\hline 40 à 49 ans & Réf. & & & & Réf. & & & \\
\hline 50 à 59 ans & $-0,14$ & & $-0,02$ & $-1,2$ & 0,00 & & 0,00 & 0,0 \\
\hline 60 à 69 ans & $-0,84$ & $* * *$ & 0,00 & 0,1 & $-0,39$ & $* *$ & 0,02 & 0,6 \\
\hline 70 ans et plus & $-1,33$ & *** & $-0,15$ & $-11,2$ & $-0,78$ & *** & $-0,32$ & $-8,3$ \\
\hline Agriculteur & $-0,39$ & & 0,04 & 2,9 & $-0,16$ & & 0,00 & 0,0 \\
\hline Chef d'entreprise & $-0,30$ & & 0,02 & 1,5 & $-0,34$ & & 0,02 & 0,5 \\
\hline Cadre supérieur & $-0,23$ & & $-0,01$ & $-0,4$ & $-0,66$ & $* * *$ & $-0,06$ & $-1,5$ \\
\hline Cadre moyen & $-0,24$ & & 0,01 & 0,6 & $-0,34$ & $* *$ & $-0,05$ & $-1,2$ \\
\hline Employé & Réf. & & & & Réf. & & & \\
\hline Ouvrier & $-0,14$ & & 0,06 & 4,0 & $-0,26$ & & 0,05 & 1,3 \\
\hline Chômeur & 0,91 & $* * *$ & 0,11 & 8,0 & 0,44 & $* * *$ & 0,15 & 3,8 \\
\hline Retraité & 0,56 & & 0,05 & 3,8 & 0,38 & $* * *$ & 0,28 & 7,2 \\
\hline Autre inactif & 1,81 & $* * *$ & 0,30 & 21,6 & 0,38 & $* * *$ & $-0,61$ & $-16,0$ \\
\hline Nul/Non déclaré & 0,06 & & $-0,02$ & $-1,2$ & $-0,18$ & $* *$ & 0,20 & 5,2 \\
\hline$C E P, B E P C, C A P, B E P$ & Réf. & & & & Réf. & & & \\
\hline Baccalauréat ou supérieur & $-0,07$ & & $-0,02$ & $-1,8$ & $-0,14$ & & $-0,17$ & $-4,5$ \\
\hline Somme des contributions & & & 0,47 & 34,4 & & & 0,19 & 4,9 \\
\hline
\end{tabular}

Note: $* * * \mathrm{p}<1 \% ; * * \mathrm{p}<5 \% ; * \mathrm{p}<10 \%$.

Ce tableau présente les risques d'être dépressif (logits) calculés pour chaque sexe en empilant les échantillons de 1970 et de 2002, puis les contributions de chaque modalité au taux de dépressifs et la part relative qui rapporte la contribution à l'écart entre les taux de dépressifs de 2002 et 1970 sont calculées. Champ : Personnes de référence et conjoint-e-s âgé-e-s d'au moins 18 ans.

Source : Enquêtes « Santé » 1970 et 2002, échantillons empilés. 


\section{ANNEXE 4. - Score de la dépression selon Duke}

Le score de la dépression selon Duke s'inscrit dans un questionnaire servant à déterminer le profil de santé des personnes grâce à dix-sept questions dont cinq concernent la dépression. Les items qui composent le volet dépressif sont :

1. Je me décourage trop facilement

2. J'ai du mal à me concentrer

3. Vous avez eu des problèmes de sommeil

4. Vous avez eu l'impression d'être vite fatigué(e)

5. Vous avez été triste ou déprimé(e)

Selon les items, trois réponses sont proposées : 1: «Pas du tout» ou «Non ce n'est pas mon cas »; 2 : «Un peu » ou «C'est à peu près mon cas »;3: « Beaucoup » ou « Oui c'est tout à fait mon cas ». Des points leur sont attribués $(0,1,2)$ et l'addition multipliée par 10 constitue le score de dépression de Duke (Guillemin et al., 1997).

\section{RÉFÉRENCES BIBLIOGRAPHIQUES}

AMAR E., BALSAN D., 2004, «Les ventes d'antidépresseurs entre 1980 et 2001 », Études et résultats, 285.

ANESHENSEL C. S., 1992, "Social stress : theory and research», Annual review of sociology, 18, p. 15-38.

Aneshensel C. S., RutTer C. M., LaChenbruch P. A., 1991, « Social structure, stress, and mental health : competing conceptual and analytic models », American sociological review, 56, 2, p. 166-178.

Anguis M., Cases C., Surault P., 2002, «L'évolution des suicides sur longue période : le rôle des effets d'âge, de date et de génération », Études et résultats, 185.

Aouba A., PÉquignot F., CAmelin L., Jougla É., 2011, «Évaluation de la qualité et amélioration de la connaissance des données de mortalité par suicide en France métropolitaine, 2006 », Bulletin épidémiologique hebdomadaire, 47-48, p. 497-500.

Aseltine R. H., Kessler R. C., 1993, "Marital disruption and depression in a community sample», Journal of health and social behavior, 34, 3, p. 237-251.

BASTIDE R., 1965, Sociologie des maladies mentales, Paris, Flammarion.

Baudelot C., Establet R., [1984] 2002, Durkheim et le suicide, Paris, Presses Universitaires de France [6 $6^{\mathrm{e}}$ éd.].

Baudelot C., Gollac M., Bessières C., Coutant I., Godechot O., Serre D., VIGUIER F., 2003, Travailler pour être heureux? Le bonheur et le travail en France, Paris, Fayard.

Beaud S., Pialoux M., 2003, Violences urbaines, violence sociale. Genèse des nouvelles classes dangereuses, Paris, Fayard.

Beck F., Guilbert P., Gautier A., 2007, Baromètre santé 2005. Attitudes et comportements de santé, Saint-Denis, INPES : http://www.inpes.sante.fr/.

BESNARD P., 1987, L'anomie: ses usages et ses fonctions dans la discipline sociologique depuis Durkheim, Paris, Presses Universitaires de France. 
BIBRING E., 1953, «The mechanisms of depression», dans P. GREENACRE (ed.), Affective disorders. Pyschoanalytic contribution to their study, New York (NY), International Universities Press, p. 13-48.

Boltanski L., Chiapello È., 1999, Le nouvel esprit du capitalisme, Paris, Gallimard.

BouRdieu P., 1994, Raisons pratiques, Paris, Le Seuil.

BowlBy J., [1984] 2008, Attachement et perte. Vol. 3 : La perte, tristesse et dépression, Paris, Presses Universitaires de France.

Cassan F., Mazuy M., Clanché F., 2001, «Refaire sa vie de couple est plus fréquent pour les hommes », Insee première, 797.

CAStel P.-H., 2013, «Folie du Vieux Monde, folie du Nouveau Monde », La vie des idées:

http://www.laviedesidees.fr/Folie-du-Vieux-Monde-folie-du.html.

Chauvel L., 1998, « La croissance du suicide et les problèmes de la société française après les Trente glorieuses », document de travail pour les $2^{e s}$ Journées nationales de prévention du suicide, Paris, OFCE.

Coster S. (DE), 2005, «Depression and law violation: gendered responses to gendered stresses », Sociological perspectives, 48, 2, p. 155-187.

COUSTEAuX A.-S., PAN KÉ SHON J.-L., 2008, « Le mal-être a-t-il un genre ? Suicide, risque suicidaire, dépression et dépendance alcoolique», Revue française de sociologie, 49, 1, p. 53-92.

Dejours C., BÈGUE F., 2009, Suicide et travail : que faire ? Paris, Presses Universitaires de France.

Demazière D., 1996, «Chômage et dynamiques identitaires », dans S. Paugam, L'exclusion, l'état des savoirs, Paris, La Découverte, p. 335-343.

Du Roy I., 2009, Orange stressé. Le management par le stress à France-Télécom, Paris, La Découverte.

DURKHEIM É., [1897] 1997, Le suicide. Étude de sociologie, Paris, Presses Universitaires de France [9e éd.].

Ehrenberg A., 1998, La fatigue d'être soi. Dépression et société, Paris, Odile Jacob.

EHRENBERG A., 2001, «De la névrose à la dépression. Remarques sur quelques changements de l'individualité contemporaine », Figures de la psychanalyse, 4, p. 25-41.

Ehrenberg A., 2010, La société du malaise, Paris, Odile Jacob.

Elias N., [1939] 1990, La dynamique de l'Occident, Paris, Presses Pocket.

Elias N., [1988] 2010, «Civilisation et psychosomatique », dans N. EliAS, Au-delà de Freud. Sociologie, psychologie, psychanalyse, texte établi et présenté par M. Joly, Paris, La Découverte, p. 113-130.

FAIRLIE R. W., 2005, « An extension of the Blinder-Oaxaca decomposition technique to logit and probit models », Journal of economic and social measurement, 30, 4, p. 305-316.

FoKKEMA T., DyKSTRA P. A., 2002, «Differences in depression between married and divorced women in the Netherlands: in search of an explanation », The Netherlands journal of social sciences, 38, 1, p. 24-47.

FREDÉn L., 1983, Aspects psychosociaux de la dépression: en sortir? Bruxelles, Pierre Mardaga.

Gaymu J., Festy P., Poulain M., Beets G., 2008, Future elderly living conditions in Europe, Paris, Ined. 
GeRstel N., RiESSMAN C. K., RosenFIELD S., 1985, « Explaining the symptomatology of separated and divorced women and men : the role of material conditions and social networks », Social forces, 64, 1, p. 84-101.

Gove W. R., 1972, « The relationship between sex roles, marital status, and mental illness », Social forces, 51, 1, p. 34-44.

Gove W. R., TUdoR J. F., 1973, « Adult sex roles and mental illness », American journal of sociology, 78, 4, p. 812-835.

Guillemin F., Paul-Dauphin A., Virion J.-M., Bouchet C., BRiancon S., 1997, «Le profil de santé de Duke : un instrument générique de mesure de qualité de vie liée à la santé », Santé publique, 1, p. 35-44.

Halbwachs M., [1930] 2002, Les causes du suicide, Paris, Presses Universitaires de France.

HalbWaChS M., 1947, «L'expression des émotions et la société », dans M. HaLBWACHS, Échanges sociologiques, Paris, Centre de Documentation Universitaire, p. 9 :

http://classiques.uqac.ca/classiques/Halbwachs_maurice/classes_morphologie/partie_2/ texte_2_4/expression_emotions.pdf.

Hawton K., HeERIngen K. (van), 2009, «Suicide», The lancet, 373, 9672, p. $1372-1381$.

Horwitz A. V., White H. R., 1991, «Becoming married, depression, and alcool problems among young adults », Journal of health and social behavior, 32, 3, p. 221-237.

Horwitz A. V., WhITE H. R., 1998, « The relationship of cohabitation and mental health : a study of young adult cohort », Journal of marriage and the family, 60, 2, p. 505-514.

Hughes M., Gove W. R., 1981, «Living alone, social integration, and mental health », American journal of sociology, 87, 1, p. 48-74.

INSEE, 2012, «L'économie française », Insee références.

JACOBSON E., 1971, Depression, New York (NY), International Press.

JANET P., 1923-1924, « La tension psychologique et ses oscillations », dans G. DuMAS (éd.), Traité de psychologie, Paris, Alcan, p. 919-952.

JANET P., 1929, L'évolution psychologique de la personnalité, Paris, A. Chahine.

JONG-GIERVELD J. (DE), TILBURG T. G. (VAN), 1987, « The partner as source of social support in problem and non-problem situations », Journal of social behaviour and personality, 2, 2, p. 191-200.

KARASEK R., THeORell T., 1990, Healthy work: stress, productivity, and the reconstruction of working life, New York (NY), Basic Books.

KAUFMANN J.-C., 1999, La femme seule et le prince charmant : enquête sur la vie en solo, Paris, Nathan.

LAMBOy B., LÉON C., Guilbert P., 2007, « Troubles dépressifs et recours aux soins dans la population française à partir des données du Baromètre Santé 2005 », Revue d'épidémiologie et de santé publique, 55, 3, p. 222-227.

Le PAPE A., Lecomte T., 1999, "Prévalence et prise en charge médicale de la dépression en 1996-1997 », Questions d'économie de la santé, 21.

Leroux I., Morin T., 2006, "Facteurs de risque des épisodes dépressifs en population générale », Études et résultats, 545.

LESTHAEGHE R., 1995, «The second demographic transition in Western countries : an interpretation », dans K. O. MASON, A.-M. JENSEN (eds.), Gender and family change in industrialized countries, Oxford, Clarendon Press, New York (NY), Oxford University Press, p. 17-62. 
MARUANi M. (dir.), 2005, Femmes, genre et sociétés: l'état des savoirs, Paris, La Découverte.

MERTON R. K., 1938, « Social structure and anomie », American sociological review, 3, 5, p. 672-682.

OCDE, 2012, Santé mentale et emploi. Mal-être au travail ? Mythes et réalités sur la santé mentale et l'emploi, Paris, OCDE.

OMs, 2008, Classification statistique internationale des maladies et des problèmes de santé connexes, Genève, OMS.

PAN KÉ SHON J.-L., 2002, «Être seul », dans Données sociales. La société française 2002-2003, Paris, Insee, p. 587-594.

PEARLIN L. I., SCHOOLER C., 1978, « The structure of coping », Journal of health and social behavior, 19, 1, p. 2-21.

RUSHING W. A., 1979, « Marital status and mental disorder: evidence in favor of a behavioral model », Social forces, 58, 2, p. 540-556.

Sapinho D., Chan-Chee C., Briffault X., Guignard R., Beck F., 2008, « Mesure de l'épisode dépressif majeur en population générale: apports et limites des outils », Bulletin épidémiologique hebdomadaire, 35-36, p. 313-317.

Sermet C., Cambois E., 2002, «Mesurer l'état de santé », dans G. Caselli, J. Vallin, G. Wunsch (dirs.), Démographie : analyse et synthèse. Vol. 3 : Les déterminants de la mortalité, Paris, Ined, p. 25-52.

Sher L., OQuendo M. A., MANN J. J., 2001, « Risk of suicide in mood disorders », Clinical neuroscience research, 1, 5, p. 337-344.

SimON R. W., 2002, « Revisiting the relationships among gender, marital status, and mental health », American journal of sociology, 107, 4, p. 1065-1096.

Singly F. (DE), 2007, Sociologie de la famille contemporaine, Paris, Armand Colin [3e éd.].

Singly F. (DE), 2011, Séparée. Vivre l'expérience de la rupture, Paris, Armand Colin.

Tocqueville A. (DE), [1840] 1951, De la démocratie en Amérique, tome 2, Paris, Éditions M.-Th. Génin, Librairie de Médicis.

TOUSIGNANT M., 1992, Les origines sociales et culturelles des troubles psychologiques, Paris, Presses Universitaires de France.

TURner R. J., TURner J. B., 1999, « Social integration and support », dans C. S. Aneshensel, J. C. Phelan (eds.), Handbook of the sociology of mental health, New York (NY), Kluwer Academic, Plenum Publishers, p. 301-320.

UMberson D., Wortman C. B., Kessler R. C, 1992, «Widowhood and depression : explaining long-term gender differences in vulnerability », Journal of health and social behavior, 33, 1, p. 10-24.

VALlin J., Meslé F., 1998, «Comment suivre l'évolution de la mortalité par cause malgré les discontinuités de la statistique ? Le cas de la France de 1925 à 1993 », dans G. PAVILLON (éd.), Enjeux des classifications internationales en santé, Paris, Inserm, p. 113-156.

VAN DE Velde C., 2004, Devenir adulte. Sociologie comparée de la jeunesse en Europe, thèse de doctorat de sociologie, Paris, Institut d'Études Politiques.

Waldron I., Hughes M. E., Brooks T. L., 1996, «Marriage protection and marriage selection. Prospective evidence for reciprocal effects of marital status and health », Social science \& medicine, 43, 1, p. 113-123. 


\section{Abstract}

\section{Thirty years of loneliness... and of depression.}

From 1970 to 2002, depression increased in France mainly due to the combined effects of the weakening of marital ties and changes in the social rules governing the interiority of the individual. An increase in the numbers of single people was the expression of this lower marital integration. The economic depression that followed the post-war boom, and social pressures to be a distinct and powerful self-occurred, simultaneously, altering the regulation of the subject. By using data on depression and suicide, and combining micro and macro approaches, it is possible to evaluate the effects of the growth of various categories of individuals on depression and to better understand the impact of tensions related to the economic crisis of the 1980s. Examination in terms of the shocks associated with the state of widowhood or unemployment leads to the hypothesis of a model of adaptation to stress, substituting a new normality for the old, rather than continuous progression.

Key words. DEPRESSION - LONELINESS - SUICIDE - PLASTICITY - ECONOMIC DEPRESSION

\section{ZUSAMMENFASSUNG}

\section{Dreißig Jahre Einsamkeit... und Depression.}

Zwischen 1970 und 2002 ist die Zahl der Depressionen in Frankreich gestiegen, besonders unter der gemeinsamen Einwirkung aus der Abschwächung der ehelichen Integration und aus den Änderungen der die Interiorität des Individuums strukturierenden Regeln. Der Anstieg der Zahl der Einzelpersonen hat zu einer Kristallisierung dieser geringeren ehelichen Integration geführt. Gleichzeitig haben die Wirtschaftskrise nach den dreißig "glorreichen " Nachkriegsjahren und die sozialen Forderungen zur Herausbildung eines unterschiedlichen und leistungsfähigen Selbst die Subjektregulierung verändert. Unter Berücksichtigung der Daten zur Depression und zum Selbstmord und mit Hilfe einer Mikro- und Makroannäherungsmethode wird es möglich, die Auswirkungen der Zunahme der verschiedenen Kategorien der Einzelpersonen auf die Depression zu bewerten und die Entwicklung der Spannungen in Verbindung mit der Wirtschaftskrise der achtziger Jahre besser zu erfassen. Die Prüfung in Begriffen von Schocks und gewöhnlichen Witwen- und Arbeitslosenzustand führt, in Bezug auf diese Spannungen, zur Hypothese eines Anpassungsmodells, das die alte Normalität durch eine neue ersetzt, statt zum Modell einer fortlaufenden Entwicklung.

Wörter Schlüssel. Depression - EinSAmkeit - Selbstmord - Plastizität - Krise 


\section{RESUMEN}

\section{Treinta años de soledad... y de depresión.}

De 1970 al 2002, la depresión ha aumenta en Francia especialmente por los efectos asociados a una disminución de la integración conyugal y a las modificaciones de las reglas que estructuran interiormente a la persona. El incremento de los efectivos diversos de personas solas ha concretizado esta menor integración conyugal. La crisis económica que siguió los Treinta Gloriosos, las exhortaciones sociales de ser alguien distinto y perfecto han intervenido simultáneamente, modificando la regulación del sujeto. Activando los datos relativos a la depresión y al suicidio, combinando los enfoques micro y macro, se puede evaluar los efectos de la depresión sobre el incremento de las diversas categorías de personas solas para mejor conocer el impacto de las tensiones que están ligadas a la crisis económica de los años 1980. El examen en términos de choques y del estado normal de la viudez o del desempleo nos lleva a la hipótesis de un modelo de adaptación a las tensiones, que substituye una nueva normalidad a la anterior, más bien que a la de una aumentación continúa.

Palabras claves. DEPRESIÓN - SOLEDAD - SUICIDIO - PLASTICIDAD - CRISIS 